\title{
Hyperbolic cylinders and entanglement entropy: gravitons, higher spins, $p$-forms
}

\author{
Justin R. David and Jyotirmoy Mukherjee \\ Centre for High Energy Physics, Indian Institute of Science, \\ C. V. Raman Avenue, Bangalore 560012, India \\ E-mail: justin@iisc.ac.in, jyotirmoym@iisc.ac.in
}

ABSTRACT: We show that the entanglement entropy of $D=4$ linearized gravitons across a sphere recently computed by Benedetti and Casini coincides with that obtained using the Kaluza-Klein tower of traceless transverse massive spin-2 fields on $S^{1} \times A d S_{3}$. The mass of the constant mode on $S^{1}$ saturates the Brietenholer-Freedman bound in $A d S_{3}$. This condition also ensures that the entanglement entropy of higher spins determined from partition functions on the hyperbolic cylinder coincides with their recent conjecture. Starting from the action of the 2-form on $S^{1} \times A d S_{5}$ and fixing gauge, we evaluate the entanglement entropy across a sphere as well as the dimensions of the corresponding twist operator. We demonstrate that the conformal dimensions of the corresponding twist operator agrees with that obtained using the expectation value of the stress tensor on the replica cone. For conformal $p$-forms in even dimensions it obeys the expected relations with the coefficients determining the 3-point function of the stress tensor of these fields.

KEYwords: Conformal Field Theory, Field Theories in Higher Dimensions, Higher Spin Gravity

ArXiv EPRINT: 2005.08402 


\section{Contents}

1 Introduction 1

2 Spherical entangling surface and hyperbolic cylinders 4

2.1 The conformal scalar 6

2.2 Free Maxwell field in $D=4 \quad 8$

3 Antisymmetric tensors $\quad 12$

3.12 -form in $D=6 \quad 12$

3.2 Conformal forms in $D$ dimensions 21

4 Symmetric tensors 23

4.1 Gravitons in $D=4 \quad 23$

$\begin{array}{ll}4.2 & \text { Higher spin fields in } D=4\end{array}$

4.3 Consistency check 28

4.4 Symmetric tensors in arbitrary $D \quad 30$

5 Conclusions $\quad 31$

$\begin{array}{ll}\text { A Change in measures } & 32\end{array}$

B Partition function of the graviton: $S^{1} \times R^{3} \quad 35$

C Brietenholer-Freedman bound for higher spins 39

D Twist weight from the replica cone: Maxwell field 40

E Rényi entropies and weights of twists 45

\section{Introduction}

Entanglement entropy of a sub-region in quantum field theories is an important quantity. For conformal field theories in 2 dimensions, the coefficient of logarithmic term is proportional to the central charge or the degrees of freedom of the system. A similar statement holds true for conformal field theories in higher dimensions. Entanglement entropy has found applications in topics that encompasses different areas of theoretical physics from black hole physics and holography to phase transitions in condensed matter theory. In spite of this, it is a difficult quantity to evaluate even for free theories. If the theory admits a gauge symmetry then one needs to define the algebra of gauge invariant observables in a sub-region to evaluate the entanglement entropy. Entanglement entropy in a theory of 
gravity or a theory with higher spin fields also suffers from this problem due to the difficulty of defining what is a subregion.

Recently Benedetti and Casini [1] evaluated the entanglement entropy across the 2sphere of free linearized gravitons in $D=4$ dimensions. This theory can be considered as a quantum field theory of spin-2 particles and it can be treated just as the Maxwell field. After fixing gauge and by canonically quantising the theory in spherical co-ordinates they obtain the following result for the logarithmic coefficient

$$
S_{\mathrm{EE}}=\frac{61}{45} \log (\epsilon)
$$

where $\epsilon$ is the dimensionless cutoff. Furthermore using the observations from the spin-2 case, they conjectured that the logarithmic coefficient for the entanglement entropy of free higher spin- $s$ particles across a sphere is given by

$$
S_{\mathrm{EE}}=\frac{1+15 s^{2}}{45} \log (\epsilon) .
$$

Setting $s=1$ in (1.2) reproduces the logarithmic coefficient of the free Maxwell field. However this contribution does not capture the edge modes [2-4] or the contribution of the electric centre [5,6]. Therefore, the entanglement entropy given in (1.1) of the gravitons also does not include these subtle modes. For the purposes of the present paper we ignore such contributions. The Maxwell theory is conformal and indeed, its logarithmic coefficient can be obtained from studying thermodynamics in deSitter space which is conformally related to causal development of the 2-sphere. Surprisingly it was noted in [7], that the logarithmic coefficients in (1.2) can also be obtained from the thermodynamics of spin- $s$ particles in deSitter space using results of earlier work [8]. This is surprising because the spin-2 or the higher spin theory is not conformal and a local stress tensor for these theories is not well defined.

In this paper we wish to understand this logarithmic coefficient for gravitons and the conjecture for the higher spin particles further. It would be satisfying if a path integral approach to evaluating entanglement entropy can be extended to these fields. For a conformal field theory in $D$ dimensions, the Rényi entropy/entanglement entropy can be obtained by evaluating the partition of the theory on the hyperbolic cylinder, $S^{1} \times A d S_{d}$ with $d+1=D[9]$. Therefore given the action of the theory on the hyperbolic cylinder one could perform the path integral and evaluate the entanglement entropy from the partition function. Though, the spin-2 theory is not conformal invariant, we wish to purse this approach to study the logarithmic coefficient for the spin-2 field.

Before we proceed, as a warm up exercise we consider the Maxwell action on $S^{1} \times A d S_{3}$. We adapt the methods of gauge fixing developed in [10-12] for this theory and show that the partition function of the Maxwell theory on $S^{1} \times A d S_{3}$ reduces to a Kaluza-Klein tower of the transverse vector on $A d S_{3}$. The mass of the constant mode on $S^{1}$ saturates the Brietenholer-Freedman bound of the spin-1 field on $A d S_{3}$, an observation which was missed earlier and which plays and important role in the higher spin generalisation. Evaluating the entanglement entropy, we find that it precisely coincides with the $s=1$ result of (1.2). We 
also evaluate the weight of the co-dimension 2 twist operator localized on the entangling surface from the partition function. This agrees with that obtained using the expectation value of the stress tensor on the replica cone.

To consolidate these observations, we also study the conformal 2-form in 6 dimensions. We start with the action of the 2 form on $S^{1} \times A d S_{5}$, fix gauge and show that the partition function reduces to that a Kaluza-Klein of tower of co-exact 2-forms on $A d S_{5}$. Indeed again, the mass of the constant mode on $S^{1}$ saturates the Brietenholer-Freedman bound. For the 2 -form we also evaluate the conformal dimensions of the co-dimension two twist operator by evaluating the expectation value of the stress tensor on the replica cone generalising the methods of [13]. The result agrees precisely with that obtained from the partition function.

The detailed study of the $\mathrm{U}(1)$ and the 2-form theory shows that the entanglement entropy and the weight of the co-dimension twist operator are integral transforms of the Plancherel measure of the corresponding field on $A d S_{5}$. We use this observation to obtain the putative entanglement of conformal $p$-forms in even dimensions to $D=14$. At every instance we perform consistency checks on the results. One non-trivial check is that the dimensions of the twist operator obey the expected relations [14] with the parameters determining the three point function of the stress tensor of the theory of conformal $p$-form.

The theory of linearized gravitons in flat space is not conformal invariant, furthermore the hyperbolic cylinder $S^{1} \times A d S_{3}$ is not a solution to the Einstein equations with or without a cosmological constant. These reasons prevent us from directly considering the theory of linearised gravitons on $S^{1} \times A d S_{3}$. We instead consider the gauge invariant partition function of linearised gravitions on $S^{1} \times R^{3}$ and introduce a principle to turn on curvature in the spatial directions. This leads us the theory of a Kaluza-Klein tower of traceless transverse spin-2 fields on $S^{1} \times A d S_{3}$. The mass of the constant mode on $S^{1}$ saturates the Brietenholer-Freedman bound. Evaluating the entanglement entropy from this partition function we see that the logarithmic coefficient precisely coincides with that in (1.1). We demonstrate that this partition function is scale invariant and depends only on the ratio of the radius of $S^{1}$ and $A d S_{3}$. We repeat the same analysis on the spin- $s$ field and obtain (1.2) for the entanglement entropy. The sphere is conformally related to the space $S^{1} \times A d S_{3}$ As a consistency check on our results we show that the difference of the entanglement entropy evaluated from the partition function of the Kaluza-Klein tower transverse spin- $s$ fields at the Brietenholer-Freedman bound and that evaluated from $S^{1} \times A d S_{3}$ and that from spin- $s$ fields precisely agrees with the edge mode partition function of spin- $s$ fields evaluated recently by [15]. This check also shows that the just as in the spin- 1 case, the hyperbolic cylinder method misses the edge mode contributions to entanglement entropy. Finally we provide an argument from the form of the Plancherel measure of spin- $s$ fields on $A d S_{3}$ why the entanglement entropy from the partition function on $S^{1} \times A d S_{3}$ must agree with that conjectured by [9].

The organisation of this paper is as follows. In section 2, after a brief review we revist the Maxwell theory on $S^{1} \times A d S_{3}$ and evaluate the R'enyi entropies and twist operator dimensions. In section 3 we consider the action of the 2 -form in $D=6$, and evaluate the gauge invariant partition function on $S^{1} \times A d S_{5}$. This is then used to evaluate the Rényi entropies and the twist operator dimensions. We show that the twist operator 
dimensions coincides with that obtained in from the expectation values of the stress tensor of the 2 -form on the cone. We use these observations and extend the analysis to conformal forms in even $D \leq 14$ dimensions. In section 4 we show that the entanglement entropy obtained from the partition function of a Kaluza-Klein tower of gravitons on $S^{1} \times R^{3}$ at the Brietenholer-Freedman bound. coincides precisely with 1.1. We then extend this to higher spin field in $D=4$. As a consistency check we show that the edge modes of the higher spin fields using our approach precisely coincides with that obtained in [15]. For completeness evaluate the entanglement entropy from higher spin partition functions to all and all even $D \leq 14$. Section 5 contains our conclusions. The appendices contain various details which are necessary for completeness and to arrive at the conclusions in the main text.

\section{Spherical entangling surface and hyperbolic cylinders}

Consider a conformal field theory in $D$-dimensions and let $\Sigma$ be the $D-2$-dimensional sphere of radius $R$ at a given time. Let the causal development of this region be denoted by $\mathcal{D}$. We briefly recall the argument put forward in [9] to evaluate the entanglement entropy obtained by tracing over the region outside the $D-2$-dimensional sphere. It was shown in [9] that the entanglement entropy of the sphere is equal to the Von-Neumann entropy of the density matrix of the vacuum on $\mathcal{D}$ which we denote by $\rho_{\mathcal{D}}$. Furthermore a conformal transformation relates the density matrix of the theory on $\mathcal{D}$ to the thermal density matrix on the hyperbolic cylinder $\mathbb{R} \times A d S_{d}$ where $d+1=D$, with temperature $T=\frac{1}{2 \pi R}$.

To be explicit, the Minkowski space is given by the metric

$$
d s^{2}=-d t^{2}+d r^{2}+r^{2} d \Omega_{D-2}^{2} .
$$

Here $d \Omega_{d-2}^{2}$ is the metric on the $D-2$ unit sphere. The entangling sphere $\Sigma$ is at radius $R$. The causal development of this sphere can be related to the hyperbolic cylinder using the following co-ordinate transformation

$$
t=R \frac{\sinh \frac{\tilde{\tau}}{R}}{\cosh u+\cosh \frac{\tilde{\tau}}{R}}, \quad r=R \frac{\sinh u}{\cosh u+\cosh \frac{\tilde{\tau}}{R}} .
$$

The Minkowski metric in (2.1) then transforms to

$$
\begin{aligned}
d s^{2} & =\hat{\Omega}^{2}\left[-\frac{d \tilde{\tau}^{2}}{R^{2}}+\left(d u^{2}+\sinh ^{2} u d \Omega_{D-2}^{2}\right)\right], \\
\hat{\Omega}^{2} & =\frac{R^{2}}{\cosh u+\cosh \frac{\tilde{\tau}}{R}} .
\end{aligned}
$$

This metric is conformal to $\mathbb{R} \times A d S_{d}$. It is convenient to define the co-ordinate

$$
\tau=\frac{\tilde{\tau}}{R} .
$$

The thermal density matrix on this space is given by

$$
\rho_{\mathcal{H}}=\frac{e^{2 \pi H_{\tau}}}{\mathcal{Z}}, \quad \mathcal{Z}=\operatorname{Tr}\left(e^{2 \pi H_{\tau}}\right) .
$$


where $H_{\tau}$ is the generator of translations along the co-ordinate $\tau$. To evaluate the partition function $\mathcal{Z}$ using the path integral, we need to identify $\tau \rightarrow \tau+2 \pi$. Since the causal development of the sphere in Minkowski space $\mathcal{D}$ is conformally equivalent to the hyperbolic cylinder we have the relation

$$
\rho_{\mathcal{D}}=U^{-1} \rho_{\mathcal{H}} U=\frac{U^{-1} e^{2 \pi H_{\tau}} U}{\mathcal{Z}} .
$$

We now use the relation between $\rho_{\mathcal{D}}$ and $\rho_{\mathcal{H}}$ to write down a formula for the Rényi entropy across the sphere $\Sigma[16,17]$. The Rényi entropy is given by

$$
S_{q}=\frac{1}{1-q} \log \operatorname{Tr}\left(\rho_{\mathcal{D}}^{q}\right)
$$

Let us define the free energies

$$
\mathcal{F}_{q}=-\log \mathcal{Z}_{q}=-\log \operatorname{Tr}\left(\rho_{\mathcal{D}}^{q}\right) .
$$

Then using (2.6) we obtain

$$
S_{q}=\frac{q \mathcal{F}_{1}-\mathcal{F}_{q}}{1-q}
$$

Therefore evaluating $\mathcal{Z}_{q}$, the path integral on the hyperbolic cylinder $S^{1} \times A d S_{d}$ with the identification $\tau \rightarrow \tau+2 \pi q$ enables us to evaluate the Rényi entropies across $\Sigma$. The entanglement entropy is given by

$$
S_{E E}=\lim _{q \rightarrow 1} S_{q}
$$

We can also obtain the conformal dimension of the co-dimension two twist operator creating the located on the entangling surface using the free energy on the hyperbolic cylinder. This is given by

$$
h_{q}=\frac{q}{d \operatorname{Vol}\left(A d S_{d}\right)}\left(\left.\partial_{q} \mathcal{F}\right|_{q=1}-\partial_{q} \mathcal{F}\right) .
$$

This formula arises from the fact that $h_{q}$ essentially captures the energy density due to the presence of the defect along the entangling surface $[14,18]$. The regulated volume of $A d S_{d}$ in given in (2.19).

The relationship between $\rho_{\mathcal{D}}$ and $\rho_{\mathcal{H}}$ is true for conformal field theories. For theories which are not conformally invariant, in general there is no such relation. The theory of free linearized gravitons in $D=4$ is not conformally invariant. Therefore we should a-priori expect no relation between the entanglement entropy across the 3 -sphere to the thermal partition function on the hyperbolic cylinder $S^{1} \times A d S_{3}$. One of the results we would like to show in this paper is that the entanglement entropy of gravitons across the 3 -sphere evaluated in [1] using canonical quantization precisely agrees with that evaluated using the partitions functions on the hyperbolic cylinder. However the gravitons in this case are massive. The mass is such that the constant mode on $S^{1}$ saturates the BrietenholerFreedmann bound on $A d S_{3}$.

In this next section we review the case of the conformal scalar in $D$ dimensions and observe that the corresponding theory on the hyperbolic cylinder saturates the BrietenholerFreedmann bound. Then we examine the Maxwell theory in $D=4$ on the hyperbolic 
cylinder perform the gauge fixing and show the Kaluza-Klein zero mode of the resulting gauge invariant vector on $A d S_{3}$ saturates its Brietenholer-Freedmann bound on $A d S_{3}$. Motivated by these observations we obtain the Brietenholer-Freedmann bound for symmetric traceless tensors of spin- $s$ in $A d S_{d}$. We proceed to evaluate the partition function of gravitons on $S^{1} \times A d S_{3}$. We show that when the Kaluza-Klein zero mode of the gauge invariant symmetric transverse spin-2 field on $A d S_{3}$ has a mass which saturates the Brietenholer-Freedmann bound then the entanglement entropy evaluated usign thermal partition functions on the hyperbolic cylinder agrees precisely with that evaluated in [1]. We use this observation and show that the entanglement entropy of higher spins in $D=4$ across the sphere evaluated by thermal partition functions on the hyperbolic cylinder using similar considerations also agrees with the conjecture made in [1].

\section{$2.1 \quad$ The conformal scalar}

In this subsection, we review the evaluation of entanglement entropy of the conformally coupled massless scalar in even $D \geq 4$ dimensions. The Weyl invariant action of the real scalar in $D$ dimensions is given by

$$
S=-\frac{1}{2} \int d^{D} x \sqrt{g}\left(\partial_{\mu} \phi \partial^{\mu} \phi+\frac{D-2}{4(D-1)} R \phi^{2}\right) .
$$

Note that the mass is due to the non-minimal coupling of the scalar to the metric. Consider the metric of the hyperbolic cylinder $S^{1} \times A d S_{d}$ which is given by

$$
d s^{2}=d \tau^{2}+d u^{2}+\sinh ^{2} u d \Omega_{D-2}^{2} .
$$

Substituting the Ricci curvature of this space given by $R=-d(d-1)$. the mass square $m_{0}^{2}$ is given by

$$
m_{0}^{2}=-\left(\frac{d-1}{2}\right)^{2}
$$

Note that the conformally coupled scalar satisfies the Brietenholer-Freedman bound in $A d S_{d}$, this observation was first made in [19]. The partition function is given by

$$
\mathcal{F}_{q}=-\frac{1}{2} \operatorname{Tr} \log \left[-\partial_{\tau}^{2}-\Delta_{(0)}+m_{0}^{2}\right]
$$

Here $\Delta_{(0)}$ is the spin-0 Laplacian on $A d S_{d}$ and $\tau$ is identified as $\tau \sim \tau+2 \pi q$. The eigenfunctions of the spin-0 Laplacian on $A d S_{d}$ satisfies the condition [20]

$$
\Delta_{(0)} \psi^{\{\lambda, u\}}=-\left[\lambda^{2}+\left(\frac{d-1}{2}\right)^{2}\right] \psi_{\lambda}^{\{\lambda, u\}},
$$

where $\{u\}$ refer to other quantum numbers on $A d S_{d}$. Substituting the Fourier decomposition of the field $\phi$ on $S^{1}$ as well as on $A d S_{d}$ we obtain

$$
\mathcal{F}_{q}=\frac{1}{2} \sum_{n=-\infty}^{\infty} \int_{0}^{\infty} d \mu^{(0)}(\lambda) \log \left(\lambda^{2}+\left(\frac{n}{q}\right)^{2}\right) .
$$


Here $\mu^{(0)}(\lambda)$ is the Plancherel measure of the real scalar on $A d S_{d}$. For odd $d \geq 3$, this is given by $[20] .^{1}$

$$
d \mu^{(0)}(\lambda)=\frac{\operatorname{Vol}\left(A d S_{d}\right)}{2^{d-1} \pi^{\frac{d}{2}} \Gamma\left(\frac{d}{2}\right)} \lambda^{2} \prod_{j=1}^{\frac{d-3}{2}}\left(j^{2}+\lambda^{2}\right)
$$

where the regulated Volume of $A d S_{d}$ is given by [16]

$$
\operatorname{Vol}\left(A d S_{d}\right)=\frac{2 \pi^{\frac{d-1}{2}}(-1)^{\frac{d+1}{2}}}{\Gamma\left(\frac{d+1}{2}\right)} \log (\epsilon)
$$

$\epsilon$ is a dimensionless cut off which regulates the volume of $A d S_{d}$. Comparing the eigenvalues of the spin-0 Laplacian in $A d S_{d}$ (2.16) and the expression in (2.15), shows that when the mass of the scalar saturates the Brietenholer-Freedman bound, the shift in the eigen-values of the spin-0 Laplacian cancels. The constant mode on $S^{1}$ has zero eigenvalue at $\lambda=0$ and the system therefore is gapless. The summation over $n$ in (2.17) can be done resulting in

$$
\mathcal{F}_{q}=\int_{0}^{\infty} d \mu^{(0)}(\lambda)\left[\log \left(1-e^{-2 \pi q \lambda}\right)+\pi q \lambda\right] .
$$

Substituting this in the expression for Rényi entropies given in (2.9) we obtain

$$
S_{q}=\frac{1}{1-q} \int_{0}^{\infty} d \mu^{(0)}(\lambda)\left[q \log \left(1-e^{-2 \pi \lambda}\right)-\log \left(1-e^{-2 \pi q \lambda}\right)\right] .
$$

As an example, considering the Plancherel measure for $D=4, d=3$ from (2.18) and substituting the regulated volume of $A d S_{3}$ from (2.19) we obtain

$$
S_{q}=\frac{(q+1)\left(q^{2}+1\right)}{360 q^{3}} \log (\epsilon), \quad S_{E E}=\frac{1}{90} \log (\epsilon)
$$

Since we know the free energy, conformal dimension of the twist operator associated with the entangling surface can be evaluated using (2.11) This results in

$$
h_{q}=\frac{q}{2^{d-1} \pi^{\frac{d}{2}-1} d \Gamma\left(\frac{d}{2}\right)} \int_{0}^{\infty} d \lambda\left(\lambda^{3} \prod_{j=1}^{\frac{d-3}{2}}\left(j^{2}+\lambda^{2}\right)\right)(\operatorname{coth} \pi \lambda-\operatorname{coth} \pi q \lambda) .
$$

Here we have substituted the Plancherel measure given in (2.18). Evaluating this expression for $D=4, d=3$ we obtain

$$
\begin{aligned}
h_{q} & =\frac{q}{6 \pi} \int_{0}^{\infty} d \lambda \lambda^{3}(\operatorname{coth} \pi \lambda-\operatorname{coth} \pi q \lambda), \\
& =\frac{q^{4}-1}{720 \pi q^{3}} .
\end{aligned}
$$

This approach of obtaining the conformal dimension using the partition function on $S^{1} \times$ $A d S_{d}$ agrees with that obtained by evaluating the expectation value of the stress tensor on the replica surface using the scalar propagator on the cone [21, 22]. Expressions for the Rényi entropies and the conformal dimension $h_{q}$ for even $D \geq 4$ can be obtained using (2.21) and (2.23). ${ }^{2}$

\footnotetext{
${ }^{1}$ We adopt the normalizaton of the measure given in [18].

${ }^{2}$ We restrict ourselves to even $D$, since the resulting integrals can be performed analytically. However such expressions also exist for odd $D$.
} 


\subsection{Free Maxwell field in $D=4$}

In this section we examine the case of the free $\mathrm{U}(1)$ theory on the hyperbolic cylinder. This theory has been studied extensively, the logarithmic coefficient of the entanglement for the free $\mathrm{U}(1)$ theory across the 2-sphere has been extracted using the expectation value of the stress tensor on de-Sitter space [22] and the conformally related hyperbolic cylinder [23]. Both these calculations rely on the gauge invariant two point function of the Maxwell field evaluate on the cone or on Rindler space first done by [13]. ${ }^{3}$ In [24] the logarithmic coefficient was extracted by showing that canonical quantization in spherical coordianates of the Maxwell field results in 2 independent massless scalars with angular momentum $l \geq 1$. Finally in [3], the partition function of the Maxwell field on $S^{1} \times A d S_{3}$ was evaluated using the heat kernel. The above methods do not capture the edge modes or the contribution of the electric centre [2-6], these modes restore the logarithmic coefficient so that it agrees with the trace anomaly. However it has been shown recently that these contributions arise only in the presence of interaction with heavy charges, see [25] for a detailed discussion. In this paper we focus on the free Maxwell field.

We evaluate the partition function of the U(1) theory on the hyperbolic cylinder $S_{1} \times$ $A d S_{3}$ by appropriately gauging fixing the theory and then use (2.9), (2.11) to evaluate the entanglement entropy/Rényi entropies as well as the conformal dimension of the twist operator. This is a warm up exercise to evaluate the partition function of gravitons on $S^{1} \times A d S_{3}$. The analysis also demonstrates the mass of the zero mode of the Maxwell field on $S^{1}$ satisfies the Brietenholer-Freedman bound, an observation which was missed earlier and which we will use subsequently. As mentioned earlier the partition function of the Maxwell theory has been evaluated earlier using the heat kernel method in [3]. However the gauge fixing method adopted here is different from that used in that paper. The method developed here allow us to generalise to higher spins as well as anti-symmetric tensors efficiently.

The Maxwell action is given by

$$
S=\frac{1}{4} \int d^{4} x \sqrt{g}\left(F_{\mu \nu} F^{\mu \nu}\right) .
$$

The action is invariant under the gauge transformation

$$
A_{\mu} \rightarrow A_{\mu}+\nabla_{\mu} \epsilon
$$

where $\epsilon$ is the gauge parameter. The background metric is given by

$$
d s^{2}=d \tau^{2}+d u^{2}+\sinh ^{2} u d \Omega_{2}^{2} .
$$

To fix the gauge we follow the methods developed by [10-12]. We start by decomposing the field as

$$
A_{\mu}=\left(A_{\tau}, A_{i}^{\perp}+\nabla_{i} \phi\right), \quad \text { where } \quad \nabla^{i} A_{i}^{\perp}=0 .
$$

\footnotetext{
${ }^{3}$ We revisit this calculation in appendix D.
} 
Here $i$, takes values in the $A d S_{3}$ direction. Under gauge transformation given in (2.26) these fields transform as

$$
A_{\tau} \rightarrow A_{\tau}+\nabla_{\tau} \epsilon, \quad A_{i}^{\perp} \rightarrow A_{i}^{\perp}, \quad \phi \rightarrow \phi+\epsilon .
$$

Substituting these change of variables, the action becomes

$$
\begin{aligned}
S=\frac{1}{2} \int d^{4} x \sqrt{g}\left[\left(\nabla_{i} A_{j}^{\perp}\right.\right. & \nabla^{i} A^{\perp j}-2 A_{i}^{\perp} A^{\perp i}+\left(\partial_{\tau} A_{i}^{\perp}\right)\left(\partial_{\tau} A^{\perp i}\right) \\
& +\left(\partial_{\tau} \nabla_{i} \phi\right)\left(\partial_{\tau} \nabla^{i} \phi\right) \\
& \left.+\left(\nabla_{i} A_{\tau}\right)\left(\nabla^{i} A_{\tau}\right)-2 \partial_{\tau} \nabla_{i} \phi \nabla^{i} A_{\tau}\right] .
\end{aligned}
$$

Here we have integrated by parts and dropped terms using the condition $\nabla^{i} A_{i}^{\perp}=0$. We have also interchanged the order of derivatives using

$$
\left[\nabla_{i}, \nabla_{j}\right] A_{l}=R_{l i j}^{k} A_{k}, \quad \quad R_{i j k l}=g_{i l} g_{j k}-g_{i k} g_{j l} .
$$

The second line follows due to the fact that $A d S_{3}$ is a maximally symmetric space with unit radius. The change of variables in (2.28) also comes with a change in measure which is given by (A.6)

$$
\mathcal{D} A_{\mu}=\left[\operatorname{det}\left(-\Delta_{(0)}\right)\right]^{1 / 2} \mathcal{D} A_{\tau} \mathcal{D} A_{i}^{\perp} \mathcal{D} \phi,
$$

where $\Delta_{(0)}$ is the spin-0 Laplacian on $A d S_{3}$. To treat the zero modes on the $S^{1}$ separately, we write this change of measure into that involving the zero modes on $S^{1}$ and the rest.

$$
\begin{aligned}
\mathcal{D} A_{\mu}= & {\left[\left(\operatorname{det}\left(-\Delta_{(0)}\right)\right)^{1 / 2} \mathcal{D} A_{\tau} \mathcal{D} A_{i}^{\perp} \mathcal{D} \phi\right]_{n \neq 0} } \\
& \times\left[\left(\operatorname{det}\left(-\Delta_{(0)}\right)\right)^{1 / 2} \mathcal{D} A_{\tau} \mathcal{D} A_{i}^{\perp} \mathcal{D} \phi\right]_{n=0} .
\end{aligned}
$$

Non-zero Kaluza-Klein modes. Let us first deal with the non-zero modes. From the gauge transformation given in (2.29), we see that we can fix the gauge $A_{\tau}=0$. This results in the Fadeev-Popov determinant ${ }^{4}$

$$
\Delta_{\mathrm{FP}}=\operatorname{det}\left(\partial_{\tau}\right)
$$

Therefore we can introduce the Fadeev-Popov determinant along with the delta function which chooses this gauge slice

$$
\mathcal{G}=\delta\left(A_{\tau}\right) \operatorname{det}\left(\partial_{\tau}\right)
$$

Note that this determinant does not vanish for the non-zero Kaluza-Klein modes. Combining all this, the partition function becomes

$$
\begin{aligned}
\mathcal{Z}_{n \neq 0}= & \int\left[\mathcal{D} A_{i}^{\perp}\left(\operatorname{det}\left(-\Delta_{(0)}\right)^{1 / 2} \operatorname{det}\left(\partial_{\tau}\right)\right]_{n \neq 0} \times\left[\frac{1}{\operatorname{det}\left(\partial_{\tau}\right)\left(\operatorname{det}\left(-\Delta_{(0)}\right)\right)^{1 / 2}}\right]_{n \neq 0}\right. \\
& \times \exp \left\{-\frac{1}{2} \int d^{4} x \sqrt{g}\left(A_{i n \neq 0}^{\perp}\left(-\partial_{\tau}^{2}-\Delta_{(1)}-2\right) A_{n \neq 0}^{\perp i}\right)\right\} .
\end{aligned}
$$

\footnotetext{
${ }^{4}$ To deal with the formal operator $\partial_{\tau}$, it is best to think in terms of Fourier modes. Then one just replaces $\partial_{\tau} \rightarrow i n$.
} 
We have integrated over $A_{\tau}$ using the delta function constraint. The first two determinants arise from the change in measure (2.33) and the Fadeev-Popov determinant (2.34). The determinants in the denominator comes from integration over the longitudinal mode $\phi$. These determinants cancel and we are left with the following

$$
\begin{aligned}
\mathcal{Z}_{n \neq 0} & =\int \mathcal{D} A_{i}^{\perp} \exp \left\{-\frac{1}{2} \int d^{4} x \sqrt{g}\left(A_{i n \neq 0}^{\perp}\left(-\partial_{\tau}^{2}-\Delta_{(1)}-2\right) A_{n \neq 0}^{\perp i}\right)\right\}, \\
& =\left[\frac{1}{\operatorname{det}\left(-\partial_{\tau}^{2}-\Delta_{(1)}-2\right)_{n \neq 0}}\right]^{\frac{1}{2}} .
\end{aligned}
$$

Here $\Delta_{(1)}$ is the spin-1 Laplacian on a transverse vector in $A d S_{3}$.

Kaluza-Klein zero modes. The Kaluza-Klein zero mode demands a separate treatment. From (2.30), we see that the action for these modes beome

$$
S_{n=0}=\frac{1}{2} \int d^{4} x \sqrt{g}\left[\left(\nabla_{i} A_{j}^{\perp} \nabla^{i} A^{\perp j}-2 A_{i}^{\perp} A^{\perp i}+\nabla_{i} A_{\tau} \nabla^{i} A_{\tau}\right)\right] .
$$

Here the Kaluza-Klein zero mode of $A_{\tau}$ along the $S^{1}$ is gauge invariant, from (2.29) it can be seen that it transforms as

$$
A_{\tau, n=0} \rightarrow A_{\tau, n=0} .
$$

The action (2.38) is independent of the longitudinal mode $\phi$ and therefore is gauge invariant under

$$
\phi_{n=0} \rightarrow \phi_{n=0}+\epsilon_{n=0} .
$$

We fix this gauge by restricting to the slice

$$
\mathcal{G}=\delta\left(\phi_{n=0}\right) .
$$

Note that the Fadeev-Popov determinant for this choice of gauge slice is unity. Using the measure for the zero modes in (2.33), the functional integral reduces to ${ }^{5}$

$$
\begin{aligned}
\mathcal{Z}_{n=0}= & \int\left[\mathcal{D} A_{i}^{\perp} \mathcal{D} A_{\tau} \operatorname{det}\left(-\Delta_{(0)}\right)\right]_{n=0} \\
& \times \exp \left\{-\frac{1}{2} \int d^{4} x \sqrt{g}\left(A_{i n=0}^{\perp}\left(-\Delta_{(1)}-2\right) A_{n=0}^{\perp i}+A_{\tau n=0}\left(-\Delta_{(0)}\right) A_{\tau n=0}\right)\right\} \\
= & {\left[\frac{1}{\operatorname{det}\left(-\Delta_{(1)}-2\right)}\right]^{\frac{1}{2}} . }
\end{aligned}
$$

It is interesting to note that for the $n \neq 0$ modes, the Jacobian in the change in measure given in (2.33) is cancelled by the integration of the longitudinal field $\phi$. However for the $n=0$ modes, the Jacobian is cancelled by the integration of $A_{\tau}$ which is gauge invariant. Combining the results from (2.37) and (2.42) we can write the complete partition function as

$$
\mathcal{Z}=\left[\frac{1}{\operatorname{det}\left(-\partial_{\tau}^{2}-\Delta_{(1)}-2\right)}\right]^{\frac{1}{2}} .
$$

\footnotetext{
${ }^{5}$ We have retained the integration as $\int d^{4} x \sqrt{g}$ which includes the integral over the $\tau$ direction. This just picks up a factor of $2 \pi q$ which cancels against the normalisation of the zero mode.
} 
The end result involves the determinant of only in the transverse vector on $A d S_{3}$ including all its Kaluza-Klein modes over $S^{1}$. The zero mode of this vector has mass square which is given by

$$
m_{s=1}^{2}=-2
$$

This mass saturates the Brietenholer-Freedman bound for a spin-1 field on $A d S_{3}$. We can now Fourier decompose the vector on $S^{1}$ and $A d S_{3}$. These observations will be used in section 4 to evaluate the partition function of fields of spins $s>1$.

The eigenvalues of the spin-1 Laplacian on transverse vector on $A d S_{3}$ is given by [20].

$$
\Delta_{(1)} \psi_{i}^{\{\lambda, u\}}=-\left(\lambda^{2}+2\right) \psi_{i}^{\{\lambda, u\}} .
$$

Substituting the decomposition of the transverse vector into Fourier modes as well as the eigenfunctions on $A d S_{3}$ into the partition function (2.43), the free energy is given

$$
\mathcal{F}_{q}=\frac{1}{2} \sum_{n=-\infty}^{\infty} \int d \mu^{(1)} \log \left(\lambda^{2}+\frac{n^{2}}{q^{2}}\right)
$$

Note that again that the shift from the eigenvalue of the spin-1 Laplacian in (2.45) cancels against the mass in (2.43). Just as in the case of the conformal boson, this ensures that the constant mode of the vector on $S^{1}$ has a gapless spectrum as $\lambda$ runs from 0 to $\infty$. The Plancherel measure for the spin-1 field is given by [20, 26]

$$
d \mu^{(1)}=\frac{\operatorname{Vol}\left(A d S_{3}\right)}{\pi^{2}}\left(\lambda^{2}+1\right) d \lambda .
$$

In this measure we have incorporated the spin dependent factor $g(s)$ which was determined by [26] using analytical continuation from the sphere $S^{3}$. For $d=3, g(s)=1$ and for $s=0$ and $g(s)=2$ for $s \geq 1$. The regularised volume of $A d S_{3}$ can be read out from (2.19) is given by

$$
\operatorname{Vol}\left(A d S_{3}\right)=2 \pi \log (\epsilon)
$$

Performing the sum over the Kaluza-Klein modes in the free energy (2.46) and substituting this into the expression for the Rényi entropy (2.9) we obtain

$$
\begin{aligned}
S_{q} & =\frac{1}{1-q} \int d \mu^{(1)}\left[q \log \left(1-e^{-2 \pi \lambda}\right)-\log \left(1-e^{-2 \pi q \lambda}\right)\right], \\
& =\frac{(q+1)\left(31 q^{2}+1\right)}{180 q^{3}} \log (\epsilon), \\
S_{1} & =\frac{16}{45} \log (\epsilon) .
\end{aligned}
$$

Finally we apply (2.11) to obtain the conformal dimension of the twist operator localised along the entangling surface. This results in

$$
\begin{aligned}
h_{q} & =\frac{q}{3 \pi} \int_{0}^{\infty} d \lambda \lambda\left(\lambda^{2}+1\right)[\operatorname{coth}(\pi \lambda)-\operatorname{coth}(\pi q \lambda)] \\
& =\frac{\left(q^{2}-1\right)\left(11 q^{2}+1\right)}{360 \pi q^{3}} .
\end{aligned}
$$


The above result agrees with that obtained by the expectation value of the stress tensor of the Maxwell field on the replica geometry. We revisit this calculation in appendix D.

One point worth emphasising is that this result for $h_{q}$ precisely agrees with the relations found in [14] between the first derivative and 2nd derivative of the $h_{q}$ with respect to $q$ and the two point and three point functions in a CFT. To verify this let us take the first derivative and the 2 nd derivate of the expression for $h_{q}$ given in (2.50), these are given by

$$
\left.\partial_{q} h_{q}\right|_{q=1}=\frac{1}{15 \pi},\left.\quad \partial_{q}^{2} h_{q}\right|_{q=1}=-\frac{4}{45 \pi} .
$$

Let the parameters determining the 3 point functions of a CFT by given by $a, b, c$ according to [27]. Then the relation found in [14] can be written as

$$
\left.\partial_{q} h_{q}\right|_{q=1}=\frac{\pi^{5}}{180}(14 a-2 b-5 c),\left.\quad \partial_{q}^{2} h_{q}\right|_{q=1}=-\frac{\pi^{5}}{90}(16 a-3 b-5 c) .
$$

For the free Maxwell field in $D=4$, the values of $a, b, c$ are given by [27]

$$
a=-\frac{2}{\pi^{6}}, \quad b=0, \quad c=-\frac{8}{\pi^{6}} .
$$

Substituting (2.53) into (2.52) we see that it precisely coincides with (2.51).

\section{$3 \quad$ Antisymmetric tensors}

In this section, we evaluate the entanglement entropy of a 2 -form in 6 dimensions across a 4-sphere. This question has been address before. In [28] the partition function of this theory on branched spheres which is conformally related to the hyperbolic cylinder was evaluated. In [29] the heat-kernel on the hyperbolic cylinder of this theory was assumed to factorize into that on $S^{1}$ and that of a co-exact 2 -form on $A d S_{5}$, this was then used to evaluate the Rényi entropy. Recall, that this theory is conformal and therefore using the argument reviewed in section 2 , we can evaluate the entanglement entropy by considering the partition function of the anti-symmetric 2-form on $S^{1} \times A d S_{5}$. Starting from the action we will fix gauge and reduce the partition function to that of a Kaluza-Klein tower of coexact 2-forms on $A d S_{5}$. Thus we prove the factorization which was assumed in [29]. The zero mode of this field is massless in $A d S_{5}$ and satisfies the Breitenlohner-Freedman bound of the 2 -form in $A d S_{5}$. We also evaluate the conformal dimension $h_{q}$ of the corresponding co-dimension 2 twist operator. We then perform a consistency check by evaluating the conformal dimension using the expectation value of the stress tensor on the cone. This generalises the methods of [13] to higher rank tensors. Finally, we will show that conformal dimensions of the twist operators localized on the entangling surface obeys the relations found in [14]. These relate the first and second derivative of $h_{q}$ to the coefficients of the three point function of the stress energy tensor of the theory. In section 3.2 we generalise the analysis to $D / 2-1$ forms in $D$-dimensions with $D=8,10,12,14$.

\subsection{2 -form in $D=6$}

The action of the 2 form is given by

$$
S=\frac{1}{12} \int d^{6} x \sqrt{g}\left(F_{\mu \nu \rho} F^{\mu \nu \rho}\right),
$$


where the metric is given by

$$
d s^{2}=d \tau^{2}+d u^{2}+\sinh ^{2} u d \Omega_{4}^{2}
$$

where $\tau$ is identified as $\tau \sim \tau+2 \pi q$, we will label the indices along the $A d S_{5}$ directions with $i, j \cdots$. The curvature of $A d S_{5}$ satisfies

$$
R_{i j k l}=g_{i l} g_{j k}-g_{i k} g_{j l}, \quad R_{i j}=-4 g_{i j}, \quad R=-20 .
$$

The field strength is defined from the gauge potential $A_{\mu \nu}$ by

$$
F_{\mu \nu \rho}=\nabla_{\mu} A_{\nu \rho}+\nabla_{\nu} A_{\rho \mu}+\nabla_{\rho} A_{\mu \nu}
$$

The action (3.1) is invariant under the gauge transformation

$$
\delta A_{\mu \nu}=\nabla_{\mu} \epsilon_{\nu}^{\perp}-\nabla_{\nu} \epsilon_{\mu}^{\perp}, \quad \nabla^{\mu} \epsilon_{\mu}^{\perp}=0
$$

Note that the longitudinal component of $\epsilon^{\mu}$ which we denote by $\tilde{\epsilon}$ does not enter the gauge transformation. This point will be important subsequently.

Following our pervious analysis, we start by decomposing the field as

$$
\begin{aligned}
& A_{\mu \nu}=\left\{A_{\tau i}, A_{i j}\right\}, \quad A_{\tau i}=A_{\tau i}^{\perp}+\nabla_{i} \chi_{\tau} \quad \nabla^{i} A_{\tau i}^{\perp}=0, \\
& A_{i j}=A_{i j}^{\perp}+\nabla_{i} \chi_{j}^{\perp}-\nabla_{j} \chi_{i}^{\perp}, \quad \nabla^{i} A_{i j}^{\perp}=0, \quad \nabla^{i} \chi_{i}^{\perp}=0 .
\end{aligned}
$$

Note that $A_{i j}^{\perp}$ is a co-closed 2-form. In terms of these variables the action becomes

$$
\begin{aligned}
S & =\int d^{6} x \sqrt{g}\left(\mathcal{L}_{1}+\mathcal{L}_{2}+\mathcal{L}_{3}\right), \\
\mathcal{L}_{1} & =\frac{1}{4}\left[\left(\nabla_{i} A_{\tau j}^{\perp}-\nabla_{j} A_{\tau i}^{\perp}\right)-2 \partial_{\tau}\left(A_{i j}^{\perp}+\nabla_{i} \chi_{j}^{\perp}-\nabla_{j} \chi_{i}^{\perp}\right)\right]\left(\nabla^{i} A^{\tau j \perp}-\nabla^{j} A^{\tau i \perp}\right), \\
\mathcal{L}_{2} & =\frac{1}{2} \chi^{i \perp} \partial_{\tau}^{2}\left(\Delta_{(1)}+4\right) \chi_{i}^{\perp}, \\
\mathcal{L}_{3} & =\frac{1}{4} A_{i j}^{\perp}\left(-\Delta_{\mathrm{HdR}}-\partial_{\tau}^{2}\right) A^{i j},
\end{aligned}
$$

where $\Delta_{\text {HdR }}$ refers to the Hodge-de Rham Laplacian, which acts on co-closed 2-forms as

$$
\Delta_{\mathrm{HdR}} A^{i j \perp}=\nabla_{k}\left(\nabla^{k} A^{i j \perp}+\nabla^{i} A^{j k \perp}+\nabla^{j} A^{k i \perp}\right),
$$

$\Delta_{(1)}$ is the Laplacian acting on transverse vectors on $A d S_{5}$. To obtain the action in (3.7) we have interchanged covariant derivatives at the cost of introducing curvature terms and then substituted for the curvature as given in (3.3). We also decompose the gauge parameter as

$$
\epsilon_{i}=\epsilon_{i}^{\perp}+\nabla_{i} \epsilon .
$$

The gauge transformation (3.5) becomes

$$
\begin{array}{lll}
\delta A_{\tau i}^{\perp}=\partial_{\tau} \epsilon_{i}^{\perp}, & \delta \chi_{\tau}=\partial_{\tau} \epsilon-\epsilon_{\tau}, \\
\delta A_{i j}^{\perp}=0, & \delta \chi_{i}^{\perp}=\epsilon_{i}^{\perp} .
\end{array}
$$


The gauge parameters $\epsilon_{\tau}, \epsilon$ are related by

$$
\partial_{\tau} \epsilon_{\tau}+\Delta_{(0)} \epsilon=0
$$

This results from the transversality condition (3.5) on the gauge parameter $\epsilon_{\mu}$. The Jacobian for the change of variables (3.6) is given by (A.17), (A.18),

$$
\begin{aligned}
\mathcal{D} A_{\mu \nu} & =\mathcal{D} A_{\tau i}^{\perp} \mathcal{D} \chi_{\tau} \mathcal{D} A_{i j}^{\perp} \mathcal{D} \chi_{i}^{\perp} \times J, \\
J & =\left[\operatorname{det}\left(\left(-\Delta_{(1)}-4\right)\left(-\Delta_{(0)}\right)\right)\right]^{\frac{1}{2}} .
\end{aligned}
$$

where $\Delta_{(1)}, \Delta_{(0)}$, is the spin-1 and spin-0 Laplacians on $A d S_{5}$. We can split this measure into that for the non-zero Kaluza-Klein modes on $S^{1}$ and zero Kaluza-Klein modes.

$$
\begin{aligned}
\mathcal{D} A_{\mu \nu}= & {\left[\mathcal{D} A_{\tau i}^{\perp} \mathcal{D} \chi_{\tau} \mathcal{D} A_{i j}^{\perp} \mathcal{D} \chi_{i}^{\perp} \times J\right]_{n \neq 0}, } \\
& \times\left[\mathcal{D} A_{\tau i}^{\perp} \mathcal{D} \chi_{\tau} \mathcal{D} A_{i j}^{\perp} \mathcal{D} \chi_{i}^{\perp} \times J\right]_{n=0} .
\end{aligned}
$$

Non-zero Kaluza-Klein modes. The gauge symmetry in (3.10) allows us to fix the gauge $A_{\tau i}^{\perp}, \chi_{\tau}=0$. Let us see this more explicitly, for non-zero Kaluza-Klein modes the gauge transformation on the field $A_{\tau i}$ becomes

$$
\delta A_{\tau i}^{\perp}=\partial_{\tau} \epsilon_{i}^{\perp}, \quad \delta \chi_{\tau}=\frac{1}{\partial_{\tau}}\left(\partial_{\tau}^{2}+\Delta_{(0)}\right) \epsilon .
$$

To obtain the second equation we have used the constraint (3.11) relating $\epsilon_{\tau}$ and $\epsilon$. It is clear choosing $\epsilon_{i}^{\perp}, \epsilon$ we can gauge away $A_{\tau i}$ for the non-zero modes. Therefore we can impose this condition using a delta function along with the corresponding Fadeev-Popov determinant in the path integral

$$
\mathcal{G}=\delta\left(A_{i \tau}^{\perp}\right) \delta\left(\chi_{\tau}\right)\left[\operatorname{det}\left(\left(\partial_{\tau}\right)^{5}\left(\frac{1}{\partial_{\tau}}\right)\left(\partial_{\tau}^{2}+\Delta_{(0)}\right)\right)\right]_{n \neq 0} .
$$

Note that since the delta function is in the co-ordinate system in which the gauge parameters are $\epsilon_{i}^{\perp}$ and $\epsilon, \tilde{\epsilon}$, we need to transform the integration over gauge group from $\epsilon_{\mu}$. This is then divided out. The change in measure is given in (A.25),

$$
\begin{aligned}
\mathcal{D} \epsilon_{i}^{\perp} \mathcal{D} \epsilon \mathcal{D} \tilde{\epsilon} & =\mathcal{D} \epsilon_{\mu} \times J^{\prime} \\
J^{\prime} & =\left[\operatorname{det}\left(\left(\partial_{\tau}^{2}+\Delta_{(0)}\right)\left(\frac{1}{\partial_{\tau}}\right)\right)_{n \neq 0}\right]^{-1}\left[\operatorname{det}\left(-\Delta_{(0)}\right)\right]^{-\frac{1}{2}}
\end{aligned}
$$

Integrating over $A_{\tau i}^{\perp}, \chi_{\tau}$ using the delta function we obtain the following action

$$
S=\int d^{6} x \sqrt{g}\left(\mathcal{L}_{2}+\mathcal{L}_{3}\right)
$$

After performing the functional integrals over $A_{i j}^{\perp}$ and $\chi_{i}^{\perp}$ we obtain the partition function

$$
\mathcal{Z}_{A_{i j}^{\perp}, \chi_{i}^{\perp}}=\left[\operatorname{det}\left(\left(-\partial_{\tau}^{2}-\Delta_{\text {HdR }}\right)\left(-\Delta_{(1)}-4\right)\left(\partial_{\tau}^{2}\right)^{5}\right)\right]_{n \neq 0}^{\frac{1}{2}} .
$$


The complete partition function is obtained by multiplying the one loop determinants along with the Fadeev-Popov determinant in (3.15), determinant of the Jacobian $J$ in (3.13) as well as $J^{\prime}$ in (3.16). This results in

$$
\mathcal{Z}_{n \neq 0}=\left[\frac{1}{\operatorname{det}\left(-\partial_{\tau}^{2}-\Delta_{\mathrm{HdR}}\right)_{n \neq 0}}\right]^{-\frac{1}{2}} .
$$

Note that, just as in the case of U(1) field and the graviton, we observe that the determinants involving the change of measure as well as the Fadeev-Popov determinant cancels in the final expression for the partition function.

Kaluza-Klein zero mode. The action in the Kaluza-Klein zero mode sector reduces to

$$
\begin{aligned}
S & =\int d^{6} x \sqrt{g}\left(\mathcal{L}_{1}^{\prime}+\mathcal{L}_{3}\right), \\
\mathcal{L}_{1}^{\prime} & =\frac{1}{4}\left(\nabla_{i} A_{\tau j}^{\perp}-\nabla_{j} A_{\tau i}^{\perp}\right)\left(\nabla^{i} A^{\tau j \perp}-\nabla^{j} A^{\tau i \perp}\right), \\
\mathcal{L}_{3} & =\frac{1}{4} A_{i j}^{\perp}\left(-\Delta_{\mathrm{HdR}}\right) A^{i j}{ }^{\perp} .
\end{aligned}
$$

The action admits the gauge symmetry

$$
\delta A_{\tau i}^{\perp}=0, \quad \delta \chi_{\tau}=-\epsilon_{\tau}, \quad \delta A_{i j}^{\perp}=0, \quad \delta \chi_{i}^{\perp}=\epsilon_{i}^{\perp} .
$$

We can fix the gauge by choosing the slice

$$
\mathcal{G}=\delta\left(\chi_{\tau}\right) \delta\left(\chi_{i}^{\perp}\right)
$$

Since this slice is in the frame where the gauge group is parameterized in $\epsilon_{i}^{\perp}, \epsilon, \epsilon_{\tau}$ we need to transform it to the parameters $\epsilon_{\mu}$. It is the volume of gauge group $\int \mathcal{D} \epsilon_{\mu}$ which is divided out. Note that for the zero modes, the condition $\nabla^{\mu} \epsilon_{\mu}=0$ in (3.11) reduces to $\nabla^{i} \epsilon_{i}=0$. The change in measure is given by (A.28).

$$
\mathcal{D} \epsilon_{\tau} \mathcal{D} \epsilon_{i}^{\perp} \mathcal{D} \epsilon=\mathcal{D} \epsilon_{\mu} J^{\prime}, \quad J^{\prime}=\left[\operatorname{det}\left(-\Delta_{(0)}\right)\right]_{n=0}^{-\frac{1}{2}} .
$$

We can now perform the integrations over $\chi_{\tau}, \chi_{i}^{\perp}$ using the delta function and then perform the integral over the gauge invariant variables $A_{\tau i}^{\perp}, A_{i j}^{\perp}$. This results in the following one loop determinant.

$$
\mathcal{Z}_{A_{\tau i}^{\perp}, A_{i j}^{\perp}}=\left[\operatorname{det}\left(\left(-\Delta_{\mathrm{HdR}}\right)\left(-\Delta_{(0)}-4\right)\right)\right]^{-\frac{1}{2}}
$$

Combining the Jacobians from the change of measure in (3.13) and that for the gauge group in (3.23) we obtain the following partition function

$$
\mathcal{Z}=\left[\frac{1}{\operatorname{det}\left(-\Delta_{\mathrm{HdR}}\right)_{n=0}}\right]^{\frac{1}{2}}
$$

Again we see a similar phenomenon, the one loop determinant of $A_{\tau i}^{\perp}$ cancels the change in measure in the zero mode sector. 
Combining the partition function for the both the non-zero Kaluza-Klein modes from (3.19) and (3.25) and the zero modes we obtain

$$
\mathcal{Z}=\left[\frac{1}{\operatorname{det}\left(-\partial_{\tau}^{2}-\Delta_{\mathrm{HdR}}\right)}\right]^{-\frac{1}{2}}
$$

In the end, the final result is again simple. It involves the one loop determinant of all Kaluza-Klein the co-closed forms on $A d S_{5}$. This was the assumption used by the authors of [29] to evaluate of the Rényi entropy of the self dual 2-form.

The eigenvalue of the Hodge-deRham Laplacian on $A d S_{d}$ on co-closed a $p$-form $u$ is given by

$$
\Delta_{\mathrm{HdR}} \psi_{i_{1} i_{2} \cdots i_{p}}^{\{\lambda, u\}}=-\left[\lambda^{2}+\left(\frac{d-1}{2}-p\right)^{2}\right] \psi_{i_{1} i_{2} \cdots i_{p}}^{\{\lambda, u\}} .
$$

The eigenvalues do not vanish and therefore they are co-exact forms. The Plancherel measure for co-exact $p$-forms on $A d S_{d}$ for odd $d$ is given by [30]

$$
\begin{aligned}
d \mu_{p-\text { form }}(\lambda) & =\frac{\hat{g}(p) \operatorname{Vol}\left(A d S_{d}\right)}{2^{d-1} \pi^{\frac{d}{2}} \Gamma\left(\frac{d}{2}\right)} \frac{1}{\lambda^{2}+\left(\frac{d-1}{2}-p\right)^{2}} \prod_{j=0}^{\frac{d-1}{2}}\left(\lambda^{2}+j^{2}\right), \\
\hat{g}(p) & =\frac{(d-1) !}{p !(d-p-1) !} .
\end{aligned}
$$

As a simple check, note that for $d=3, p=1$ the measure reduces to that of the vector given in (2.47).

Let us now apply these results to the case of the 2 -form on $S^{1} \times A d S_{5}$. We can use the decomposition of the co-closed form into eigenfunctions on $A d S_{5}$ and the Kaluza-Klein modes on $S^{1}$ to write the free energy as

$$
\mathcal{F}=\frac{1}{2} \sum_{n} \int d \mu_{2-\text { form }}(\lambda) \log \left(\lambda^{2}+\frac{n^{2}}{q^{2}}\right) .
$$

Here we have used the expression for the eigenvalues of the Hodge deRham Laplacian given in (3.27). The Plancherel measure is given by

$$
d \mu_{2-\text { form }}(\lambda)=\frac{1}{2 \pi^{3}} \operatorname{Vol}\left(A d S_{5}\right)\left(\lambda^{2}+1\right)\left(\lambda^{2}+4\right) .
$$

Before going ahead, let us examine if the constant mode of the 2 form along the $S^{1}$ satisfies the Breitenlohner-Freedman bound in $A d S_{5}$. Given a massive $p$-form in $A d S_{d}$, the Breitenlohner-Freedman bound is given by $[31]^{6}$

$$
m^{2} \geq-\left(p-\frac{d-1}{2}\right)^{2}
$$

Therefore a massless $\frac{d-1}{2}$ from in $A d S_{d}$ always saturates the Breitenlohner-Freedman bound. Note that the constant mode along the $S^{1}$ of the 2 -form massless in $A d S_{5}$. The

\footnotetext{
${ }^{6}$ We can use the mass-conformal dimension of a $p$-form in [31], $\Delta=\frac{d}{2}+\sqrt{m^{2}+\left(p-\frac{d-1}{2}\right)^{2}}$ to read out Breitenlohner-Freedman bound.
} 
equations of motion it satisfies is $\Delta_{\mathrm{HdR}} A_{i j}^{\perp}=0$, the masses arises only from the curvature couplings. Therefore the constant mode of the conformal 2-form in $S^{1} \times A d S_{5}$ saturates the Breitenlohner-Freedman bound. Now using (2.9) we are led to the following integral for the Rényi entropy

$$
\begin{aligned}
S_{q} & =\frac{1}{1-q} \int d \mu_{2-\text { form }}(\lambda)\left[q \log \left(1-e^{-2 \pi \lambda}\right)-\log \left(1-e^{-2 \pi q \lambda}\right)\right], \\
& =-\frac{(q+1)\left(877 q^{4}+37 q^{2}+2\right)}{5040 q^{5}} \log \epsilon .
\end{aligned}
$$

The entanglement entropy is given by

$$
S_{1}=-\frac{229}{630} \log \epsilon
$$

Similarly we use (2.11) to evaluate the conformal dimension of the twist operator corresponding to the spherical entangling surface. We obtain

$$
\begin{aligned}
h_{q} & =\frac{q}{10 \pi^{2}} \int_{0}^{\infty} d \lambda \lambda\left(\lambda^{2}+1\right)\left(\lambda^{2}+4\right)(\operatorname{coth} \pi \lambda-\operatorname{coth} \pi q \lambda), \\
& =\frac{\left(q^{2}-1\right)\left(191 q^{4}+23 q^{2}+2\right)}{5040 \pi^{2} q^{5}} .
\end{aligned}
$$

The result in (3.32), (3.33), (3.34). misses the contribution of the edge modes at the boundary of the entangling surface, that shift the result so that it agrees with the conformal anomaly. Perhaps just as in the discusion for the U(1) theory in [25], the evaluation of the entanglement entropy using the partition function on $S^{1} \times A d S_{5}$ is the result for the free 2-form theory and not for that in presence of 2-form charges. We proceed to evaluate $h_{q}$ using the expectation value of the stress tensor on the conical geometry and show that it precisely coincides with the above value. This provides us a check of our path integral methods in particular the gauge fixing procedure we adopted.

Twist operator weights from the replica cone. In a conformal field theory, the conformal dimension associated with the twist operator localized on the spherical entangling surface is the same as that of the twist operator $\sigma_{q}$ creating a co-dimension 2 planar defect or a planar entangling surface since these surfaces can be conformally mapped to each other. By conformal invariance the expectation value of the stress tensor on a planar defect completely determined by the dimension of the twist operator. To be specific let us suppose at the time slice $x^{1}=0$, the planar entangling surface is at $x^{2}=0$. We label the coordinates $a, b$. let all the rest of the longitudinal directions by labeled by $x^{i}, i=3, \cdots D$ and $r=\sqrt{\left(x^{1}\right)^{2}+\left(x^{2}\right)^{2}}$ be the transverse distance from the defect. Then the expectation value of the stress tensor takes the form

$$
\begin{aligned}
\left\langle T_{i j} \sigma_{q}\right\rangle & =-\frac{h_{q}}{2 \pi} \frac{\delta_{i j}}{r^{D}}, & \left\langle T_{i a} \sigma_{q}\right\rangle & =0, \\
\left\langle T_{a b} \sigma_{q}\right\rangle & =\frac{h_{q}}{2 \pi} \frac{(D-1) \delta_{a b}-D n_{a} n_{b}}{r^{D}}, & n^{a} & =\frac{x^{a}}{r} .
\end{aligned}
$$

To evaluate these expectation values we can adopt the replica trick. We consider the path integral on $q$ copies of the CFT is glued along the cut $x^{2}>0$. When one uses polar 
coordinates $r, \theta$ to describe the transverse directions $x^{1}, x^{2}$, the CFT is then on a cone, in which $\theta$ is identified with the periodicity $\theta \sim \theta+2 \pi q$. We can then evaluate the expectation values of the stress tensor using propagators on this cone by the point split method. We adopt this procedure for the stress tensor of the 2 form in $D=6 .^{7}$

The stress tensor can be derived from the action given in (3.1). It is given by

$$
\begin{aligned}
T_{\mu \nu} & =\frac{1}{2} F_{\mu \rho \sigma} F_{\nu}^{\rho \sigma}-\frac{\delta_{\mu \nu}}{12} F_{\rho \sigma \delta} F^{\rho \sigma \delta}, \\
F_{\mu \nu \rho} & =\partial_{\mu} A_{\nu \rho}+\partial_{\nu} A_{\rho \mu}+\partial_{\rho} A_{\mu \nu} .
\end{aligned}
$$

We should first fix gauge and then write down the propagator of the 2 -form consistent with this gauge. The 2 form admits the gauge transformation

$$
A_{\mu \nu} \rightarrow A_{\mu \nu}+\partial_{\mu} \epsilon_{\nu}^{\perp}-\partial_{\nu} \epsilon_{\mu}^{\perp}
$$

Note that the longitudinal component of the parameter $\epsilon_{\mu}$ does not contribute to the gauge transformation. Using this symmetry we can fix the gauge

$$
\partial^{\mu} A_{\mu \nu}=0
$$

Therefore the equation of motion is given by

$$
\square A_{\mu \nu}=0 .
$$

This still allows us the gauge transformation

$$
A_{\mu \nu}^{\prime}=A_{\mu \nu}+\partial_{\mu} \epsilon_{\nu}^{\perp}-\partial_{\nu} \epsilon_{\mu}^{\perp}, \quad \text { with, } \quad \square \epsilon_{\mu}^{\perp}=0 .
$$

Then given a 2-form which satisfies the gauge condition (3.38) and (3.39) we can make a further gauge transformation so that the 2 -form in the new frame satisfies the condition

$$
\partial^{a} A_{a \mu}^{\prime}=0, \quad \partial^{i} A_{i \mu}^{\prime}=0 \quad a \in\{1,2\}, i \in\{3,4,5,6\} .
$$

To do this, choose the gauge parameter as ${ }^{8}$

$$
\epsilon_{\mu}^{\perp}=-\frac{\partial^{i} A_{i \mu}}{\widetilde{\nabla}^{2}}, \quad \hat{\nabla}^{2}=\sum_{i=3}^{6} \partial_{i}^{2} .
$$

It is easy to see that using this additional gauge transformation the 2-form satisfies the more restrictive condition (3.41). The gauge parameter also satisfies $\square \epsilon_{\mu}^{\perp}=0$.

Since we need to work on the replica cone it is convenient to use the polar coordinates

$$
d s^{2}=d r^{2}+r^{2} d \theta^{2}+\sum_{i=3}^{6} d x^{i} d x^{i}, \quad \theta \sim \theta+2 \pi q .
$$

\footnotetext{
${ }^{7}$ Appendix revisits this calculation for the $\mathrm{U}(1)$ theory in $D=4$.

${ }^{8}$ This equation and similar subsequent equations seem formal. It can be concretely understood in terms of the Fourier space in the 3,4,5,6 directions. The theory has translational symmetry in these directions.
} 
Let us define

$$
\hat{G}_{\mu \nu}^{\mu^{\prime} \nu^{\prime}}\left(x, x^{\prime}\right)=\left\langle A_{\mu \nu}(x) A^{\mu^{\prime} \nu^{\prime}}\left(x^{\prime}\right)\right\rangle .
$$

Then the Green's function of the 2 form satisfying the gauge condition (3.41) is given by

$$
\begin{aligned}
& \tilde{G}_{a b}^{a^{\prime} b^{\prime}}\left(x, x^{\prime}\right)=\tilde{G}_{a b}^{i j}\left(x, x^{\prime}\right)=\tilde{G}_{i j}^{a^{\prime} b^{\prime}}\left(x, x^{\prime}\right)=0, \\
& \tilde{G}_{a i}^{b^{\prime} j^{\prime}}\left(x, x^{\prime}\right)=-\left[\left(\frac{-P_{a} P^{b \prime}}{\widehat{\nabla}^{2}}\right)\left(\delta_{i}^{j}-\frac{\partial_{i} \partial^{j}}{\widehat{\nabla}^{2}}\right)\right] \tilde{G}\left(x, x^{\prime}\right), \quad \hat{\nabla}^{2}=\sum_{i=3}^{6} \partial_{i}^{2}, \\
& \tilde{G}_{a i}^{b^{\prime} j^{\prime}}\left(x, x^{\prime}\right)=-\tilde{G}_{i a}^{b^{\prime} j^{\prime}}\left(x, x^{\prime}\right)=-\tilde{G}_{a i}^{j^{\prime} b^{\prime}}\left(x, x^{\prime}\right)=\tilde{G}_{i a}^{j^{\prime} b^{\prime}}\left(x, x^{\prime}\right), \\
& \tilde{G}_{i j}{ }^{k^{\prime} l^{\prime}}\left(x, x^{\prime}\right)=-\left[\left(\delta_{i k}-\frac{\partial_{i} \partial^{k}}{\widehat{\nabla}^{2}}\right)\left(\delta_{j l}-\frac{\partial_{j} \partial^{l}}{\widehat{\nabla}^{2}}\right)-\left(\delta_{i l}-\frac{\partial_{i} \partial^{l}}{\widehat{\nabla}^{2}}\right)\left(\delta_{j k}-\frac{\partial_{j} \partial^{k}}{\widehat{\nabla}^{2}}\right)\right] \tilde{G}\left(x, x^{\prime}\right) .
\end{aligned}
$$

where $\tilde{G}\left(x, x^{\prime}\right)$ is the Green's function of a massless scalar on the cone. This can be found in many works, see [32] for a recent reference.

$$
\begin{aligned}
\tilde{G}\left(x, x^{\prime}\right) & =\frac{f_{q}\left(a, \theta, \theta^{\prime}\right)+g_{q}\left(a, \theta, \theta^{\prime}\right)}{4 \pi^{3} q^{2}\left(r r^{\prime}\right)^{2}\left(a-a^{-1)}\right)^{2}}, \\
\frac{a}{1+a^{2}} & =\frac{r r^{\prime}}{\left(x-x^{\prime}\right)^{i}\left(x-x^{\prime}\right)_{i}+r^{2}+r^{\prime 2}}, \\
f_{q}\left(a, \theta, \theta^{\prime}\right) & =\frac{2\left(\left(a^{\frac{1}{q}}+a^{-\frac{1}{q}}\right) \cos \left(\frac{\theta-\theta^{\prime}}{q}\right)-2\right)}{\left(a^{\frac{1}{q}}+a^{-\frac{1}{q}}-2 \cos \left(\frac{\theta-\theta^{\prime}}{q}\right)\right)^{2}}, \\
g_{q}\left(a, \theta, \theta^{\prime}\right) & =\frac{\left(a+a^{-1}\right)\left(a^{\frac{1}{q}}-a^{-\frac{1}{q}}\right)}{\left(a^{\frac{1}{q}}+a^{-\frac{1}{q}}-2 \cos \left(\frac{\theta-\theta^{\prime}}{q}\right)\right)\left(a-a^{-1}\right)} .
\end{aligned}
$$

and $P_{a}$ are defined as

$$
P_{a}=\epsilon_{a b} g^{b c} \nabla_{c}, \quad \epsilon_{12}=-\epsilon_{21}=r, \epsilon_{11}=\epsilon_{22}=0 .
$$

Note that the first line of (3.45) follows from the gauge condition $\nabla^{a} \tilde{G}_{a \mu}^{\rho^{\prime} \sigma^{\prime}}=0$ together with the anti-symmetry property of the Green's function.

To evaluate the expectation value of the stress tensor on the replica cone, we take the expression of the stress tensor given in (3.36) and separate the two points ,and then take the expectation values, using the Green's function in (3.45) finally take the coincident limit. Therefore we have

$$
\left\langle T_{\mu \nu} \sigma_{q}\right\rangle=\lim _{x \rightarrow x^{\prime}} \frac{1}{2}\left\langle F_{\mu \rho \sigma}(x) F_{\nu}^{\rho \sigma}\left(x^{\prime}\right)\right\rangle-\frac{\delta_{\mu \nu}}{12}\left\langle F_{\rho \sigma \delta}(x) F^{\rho \sigma \delta}\left(x^{\prime}\right)\right\rangle .
$$

On taking the coincident limit there are short distance divergences which are identical to that seen on using the propagator with $q=1$. To regulate these divergence we replace the propagator by the regulated propagator given by

$$
G_{\mu \nu}^{\mu^{\prime} \nu^{\prime}}\left(x, x^{\prime}\right)=\left.\tilde{G}_{\mu \nu}^{\mu^{\prime} \nu^{\prime}}\left(x, x^{\prime}\right)\right|_{q}-\left.\tilde{G}_{\mu \nu}^{\mu^{\prime} \nu^{\prime}}\left(x, x^{\prime}\right)\right|_{q=1} .
$$


For example performing these steps on the first term in the stress tensor we obtain

$$
\begin{aligned}
\left\langle F_{a \mu \nu}(x)\right. & \left.F^{b^{\prime} \mu^{\prime} \nu^{\prime}}\left(x^{\prime}\right)\right\rangle \\
= & \left(\nabla_{a} \nabla^{b^{\prime}} G_{\mu \nu}^{\mu^{\prime} \nu^{\prime}}+\nabla_{a} \nabla^{\mu^{\prime}} G_{\mu \nu}^{\nu^{\prime} b^{\prime}}+\nabla_{a} \nabla^{\nu^{\prime}} G_{\mu \nu}^{b^{\prime} \mu^{\prime}}\right) \\
& +\left(\nabla_{\mu} \nabla^{b^{\prime}} G_{\nu a}^{\mu^{\prime} \nu^{\prime}}+\nabla_{\mu} \nabla^{\mu^{\prime}} G_{\nu a}^{\nu^{\prime} b^{\prime}}+\nabla_{\mu} \nabla^{\nu^{\prime}} G_{\nu}^{b^{\prime} \mu^{\prime}}\right) \\
& +\left(\nabla_{\nu} \nabla^{b^{\prime}} G_{a \mu}^{\mu^{\prime} \nu^{\prime}}+\nabla_{\nu} \nabla^{\mu^{\prime}} G_{a \mu}^{\nu^{\prime} b^{\prime}}+\nabla_{\nu} \nabla^{\nu^{\prime}} G_{a \mu}^{b^{\prime} \mu^{\prime}}\right) \\
= & {\left[-6 \nabla_{a} \nabla^{b^{\prime}}+6 \frac{\left(\nabla_{a} P_{c}-\nabla_{c} P_{a}\right)}{\hat{\nabla}^{2}} \frac{\left(\nabla^{c^{\prime}} P^{b^{\prime}}-\nabla^{b^{\prime}} P^{c^{\prime}}\right)}{\hat{\nabla}^{2}}+6 P_{a} P^{b^{\prime}}\right] G\left(x, x^{\prime}\right) . }
\end{aligned}
$$

To arrive at the last line in the above equation, we have used similar manipulations and several identities listed in appendix D. We define

$$
G\left(x, x^{\prime}\right)=\left.\tilde{G}\left(x, x^{\prime}\right)\right|_{q}-\left.\tilde{G}\left(x, x^{\prime}\right)\right|_{q=1} .
$$

Choosing specific values for the $a, b$ indices, the result for the non-trivial components along the transverse direction of the defect are given by

$$
\begin{aligned}
\left\langle F_{\theta \mu \nu} F^{\theta^{\prime} \mu^{\prime} \nu^{\prime}}\right\rangle & =\left[-\frac{6}{r^{2}} \frac{\partial^{2}}{\partial \theta \partial \theta^{\prime}}+6 \frac{\partial^{2}}{\partial r \partial r^{\prime}}-6 \widehat{\nabla}^{2}\right] G\left(x, x^{\prime}\right), \\
\left\langle F_{r \mu \nu} F^{r^{\prime} \mu^{\prime} \nu^{\prime}}\right\rangle & =\left[\frac{6}{r^{2}} \frac{\partial^{2}}{\partial \theta \partial \theta^{\prime}}-6 \frac{\partial^{2}}{\partial r \partial r^{\prime}}-6 \widehat{\nabla}^{2}\right] G\left(x, x^{\prime}\right) .
\end{aligned}
$$

Similarly going through the analysis to evaluate the non-zero components along the transverse directions we obtain

$$
\left\langle F_{i \mu \nu} F^{j^{\prime} \alpha^{\prime} \beta^{\prime}}\right\rangle=3 \delta_{i}^{j} \widehat{\nabla}^{2} G\left(x, x^{\prime}\right) .
$$

Combining (3.52) and (3.53 we get

$$
\left\langle F_{\mu \nu \rho} F^{\mu^{\prime} \nu^{\prime} \rho^{\prime}}\right\rangle=0 .
$$

Therefore the components of the stress tensor are given by

$$
\begin{aligned}
\left\langle T_{\theta}^{\theta} \sigma_{q}\right\rangle & =\lim _{x \rightarrow x^{\prime}} \frac{1}{2}\left\langle F_{\theta \mu \nu} F^{\theta^{\prime} \mu^{\prime} \nu^{\prime}}\right\rangle \\
& =\lim _{x \rightarrow x^{\prime}}\left[-\frac{3}{r^{2}} \frac{\partial^{2}}{\partial \theta \partial \theta^{\prime}}+3 \frac{\partial^{2}}{\partial r \partial r^{\prime}}-3 \widehat{\nabla}^{2}\right] G\left(x, x^{\prime}\right), \\
& =\frac{\left(q^{2}-1\right)\left(191 q^{4}+23 q^{2}+2\right)}{1008 \pi^{2} q^{6} r^{6}} .
\end{aligned}
$$

Proceeding along the same lines we obtain

$$
\begin{aligned}
\left\langle T_{r}^{r} \sigma_{q}\right\rangle & =\lim _{x \rightarrow x^{\prime}}\left[\frac{3}{r^{2}} \frac{\partial^{2}}{\partial \theta \partial \theta^{\prime}}-3 \frac{\partial^{2}}{\partial r \partial r^{\prime}}-3 \widehat{\nabla}^{2}\right] G\left(x, x^{\prime}\right), \\
& =\frac{-191 q^{6}+168 q^{4}+21 q^{2}+2}{5040 \pi^{2} q^{6} r^{6}} \\
\left\langle T_{i}^{i} \sigma_{q}\right\rangle & =\lim _{x \rightarrow x^{\prime}} \frac{3}{2} \delta_{i}^{j} \widehat{\nabla}^{2} G\left(x, x^{\prime}\right) \\
& =-\frac{\left(q^{2}-1\right)\left(191 q^{4}+23 q^{2}+2\right)}{5040 \pi^{2} q^{6} r^{6}} .
\end{aligned}
$$


To evaluate $h_{q}$ and also to confirm the form for the expectation value of the stress tensor given in (3.35), we convert back to cartesian coordinates by setting $x^{1}=r \cos \theta, x^{2}=r \sin \theta$ and this transformation on the stress tensors in (3.55) and (3.56) to obtain

$$
\begin{aligned}
\left\langle T_{11} \sigma_{q}\right\rangle & =\frac{\left(q^{2}-1\right)\left(191 q^{4}+23 q^{2}+2\right)\left[\left(x^{1}\right)^{2}-5\left(x^{2}\right)^{2}\right]}{5040 \pi^{2} q^{6} r^{8}}, \\
\left\langle T_{22} \sigma_{q}\right\rangle & =\frac{\left(q^{2}-1\right)\left(191 q^{4}+23 q^{2}+2\right)\left[-5\left(x^{1}\right)^{2}+\left(x^{2}\right)^{2}\right]}{5040 \pi^{2} q^{6} r^{8}} \\
\left\langle T_{12} \sigma_{q}\right\rangle & =\frac{\left(-191 q^{6}+168 q^{4}+21 q^{2}+2\right) x^{1} x^{2}}{840 \pi^{2} q^{6} r^{8}}
\end{aligned}
$$

From the above equations and also the component $T_{i}^{i}$ in (3.56), we read out

$$
h_{q}=\frac{\left(q^{2}-1\right)\left(191 q^{4}+23 q^{2}+2\right)}{5040 \pi^{2} q^{5}} .
$$

This value coincides with the result obtained using the partition function on $S^{1} \times A d S_{5}$ in (3.34). Let us also obtain the first and second derivatives of $h_{q}$

$$
\left.h_{q}^{\prime}\right|_{q=1}=\frac{3}{35 \pi^{2}},\left.\quad h_{q}^{\prime \prime}\right|_{q=1}=-\frac{9}{70 \pi^{2}} .
$$

\subsection{Conformal forms in $D$ dimensions}

Our analysis of the 2 form in $D=6$ shows that the gauge invariant partition function on $S^{1} \times A d S_{5}$ reduces to the partition function of a Kaluza-Klein tower of co-exact 2 forms on $A d S_{5}$. This just depends only on the Plancherel measure of co-exact forms. In this section we use this observation and evaluate the entanglement entropies as well as twist operator weights for conformal forms in even $D$ dimensions.

The Rényi entropies are given by the expression

$$
S_{q}=\frac{1}{1-q} \int d \mu_{\frac{d-1}{2}-\text { form }}(\lambda)\left[q \log \left(1-e^{-2 \pi \lambda}\right)-\log \left(1-e^{-2 \pi q \lambda}\right)\right],
$$

where the Plancherel measure of the $(d-1) / 2$-form on $A d S_{d}$ is given by

$$
\begin{aligned}
d \mu_{\frac{d-1}{2}-\text { form }}(\lambda) & =\frac{\hat{g}(d) \operatorname{Vol}\left(A d S_{d}\right)}{2^{d-1} \pi^{\frac{d}{2}} \Gamma\left(\frac{d}{2}\right)} \prod_{j=1}^{\frac{d-1}{2}}\left(\lambda^{2}+j^{2}\right), \\
\hat{g}(d) & =\frac{(d-1) !}{\left(\Gamma\left(\frac{d+1}{2}\right)\right)^{2}} .
\end{aligned}
$$

The weight of the twist operator is given by

$$
h_{q}=\frac{q \hat{g}(d)}{2^{d-1} \pi^{\frac{d}{2}-1} d \Gamma\left(\frac{d}{2}\right)} \int_{0}^{\infty} d \lambda \lambda \prod_{j=1}^{\frac{d-1}{2}}\left(\lambda^{2}+j^{2}\right)(\operatorname{coth} \pi \lambda-\operatorname{coth} \pi q \lambda) .
$$

Appendix E lists the Rényi entropies and $h_{q}$ for all even dimensions up to $4 \leq D \leq 14$. In table 1 we list the entanglement entropies, the first and second derivatives of $h_{q}$ for these dimensions. 


\begin{tabular}{|c|c|c|c|}
\hline$D$ & Coefficient of $\log (\epsilon)$ & $\left.h_{q}^{\prime}\right|_{q=1}$ & $\left.h_{q}^{\prime \prime}\right|_{q=1}$ \\
\hline 4 & $\frac{16}{45}$ & $\frac{1}{15 \pi}$ & $-\frac{4}{45 \pi}$ \\
6 & $-\frac{229}{630}$ & $\frac{3}{35 \pi^{2}}$ & $-\frac{9}{70 \pi^{2}}$ \\
8 & $\frac{1042}{2835}$ & $\frac{4}{21 \pi^{3}}$ & $-\frac{32}{105 \pi^{3}}$ \\
10 & $-\frac{276929}{748440}$ & $\frac{20}{33 \pi^{4}}$ & $-\frac{100}{99 \pi^{4}}$ \\
12 & $\frac{45201643}{121621500}$ & $\frac{360}{143 \pi^{5}}$ & $-\frac{4320}{1001 \pi^{5}}$ \\
14 & $-\frac{108829363}{291891600}$ & $\frac{168}{13 \pi^{6}}$ & $-\frac{294}{13 \pi^{6}}$ \\
\hline
\end{tabular}

Table 1. Entanglement entropies conformal $\frac{D}{2}-1$-forms. The derivatives of $h_{q}$ agree with 3.65 obtained using conformal invariance.

$h_{q}$ and the 3 point function of the stress tensor. Using conformal invariance it was shown in [14], that the first and second derivatives of the conformal dimension of the twist operator $h_{q}$ with respect to $q$ at $q=1$ are related to the parameters determining the 2 and 3 point functions of the stress tensor. This relation is known to be violated for scalars due to total derivative terms in the stress tensor $[18,33]$. In section 2.2 we have seen that these relations hold for the Maxwell field. Therefore it is interesting to verify if such relations exist for the 2 form.

Before we proceed let us present the general relations in arbitrary $D$ dimensions. The first and second derivatives of $h_{q}$ are expected to obey the relations

$$
\begin{aligned}
\left.h_{q}^{\prime}\right|_{q=1} & =\frac{\pi^{\frac{D+3}{2}} C_{T}}{2^{D-3} D\left(D^{2}-1\right) \Gamma\left(\frac{D-1}{2}\right)}, \\
C_{T} & =\frac{\left(8 \pi^{D / 2}\right)(a(D-2)(D+3)-2 b-c(D+1))}{D(D+2) \Gamma\left(\frac{D}{2}\right)}, \\
\left.h_{q}^{\prime \prime}\right|_{q=1} & =-\frac{16 \pi^{D+1}}{D^{2} \Gamma(D+3)}\left[2 a\left(3 D^{2}-3 D-4\right)(D-2)-2 b D(D-1)-c(3 D-4)(D+1)\right],
\end{aligned}
$$

where $a, b, c$ are the parameters determining the 3 point functions of the stress tensor [27]. For the theory of free $\frac{D}{2}-1$-form in $D$ dimensions, the values of $a, b, c$ were evaluated in [34]. These are given by

$$
\begin{aligned}
& a=-\frac{D^{3} \Gamma(D-1) \Gamma\left(\frac{D}{2}\right)}{64(D-3) \pi^{\frac{3 D}{2}}}, \quad b=-\frac{(D-4) D^{3} \pi^{-\frac{3 D}{2}} \Gamma(D-1) \Gamma\left(\frac{D}{2}\right)}{64(D-3)}, \\
& c=-\frac{(D-2) D^{3} \pi^{-\frac{3 D}{2}} \Gamma(D-1) \Gamma\left(\frac{D}{2}\right)}{32(D-3)} .
\end{aligned}
$$

Now substituting these in the expressions for the derivatives of $h_{q}$ in (3.63) we obtain the following predictions for the behaviour of conformal dimensions of twist operators of the 
$\frac{D}{2}-1$ forms

$$
\left.h_{q}^{\prime}\right|_{q=1}=\frac{D \Gamma(D-1)}{2^{D+2} \pi^{\frac{D-3}{2}} \Gamma\left(\frac{D+3}{2}\right)},\left.\quad h_{q}^{\prime \prime}\right|_{q=1}=-\frac{D^{3} \Gamma(D-1) \Gamma\left(\frac{D}{2}\right)}{2 \pi^{\frac{D}{2}-1} \Gamma(D+3)} .
$$

In the $3 \mathrm{rd}$ and 4 th column of table 1 we have evaluated these derivatives directly from the evaluation of $h_{q}$ using the expression in (3.62) which relies on the partition function of conformal forms on hyperbolic cylinders. These values precisely agree with that obtained using conformal invariance in (3.65).

\section{Symmetric tensors}

In this section we evaluate the partition function of symmetric tensors of rank- $s$ on hyperbolic cylinders. From the analysis of the Maxwell field and 2-form in $D=6$ in the previous sections, it wold be natural to consider the partition function of a Kaluza-Klein tower of massive transverse symmetric traceless spin-2 fields on $A d S_{3}$. The mass of the zero mode on $S^{1}$ should saturate the Breitenlohner-Freedman bound. However since the action of the spin-2 field is not conformal invariant, it is certainly not expected such a partition function will indeed capture the entanglement entropy of the spin-2 field as evaluated by [1]. In this section we evaluate this partition function and provide arguments to support that this is indeed the natural partition function to consider and argue why the result of this partition function should coincide with the result for the entanglement entropy for the spin-2 field obtained by [1]. We generalise this discussion for the spin- $s$ field in $D=4$. Using the recent results for the edge modes for the partition function of spin- $s$ fields on spheres found by [15], we show that the result for the entanglement entropy evaluated from the partition function of the tower of Kaluza-Klein modes of spin- $s$ fields on $S^{1} \times A d S_{3}$ together with the contribution of the edge modes precisely agrees with that evaluated using the partition function of the spin- $s$ field on $S^{4}$. This agreement provides a consistency check for using the Kaluza-Klein tower of massive traceless-transverse spin- $s$ field to evaluate entanglement entropy across a spherical entangling surface.

Finally for completeness and future reference we generalise these calculations to $D=$ $6,8,10,12$.

\subsection{Gravitons in $D=4$}

The linearised action of gravitons in flat space is given by

$$
S=\int d^{4} x\left[-\partial_{\mu} h^{\mu \nu} \partial_{\alpha} h_{\nu}^{\alpha}+\frac{1}{2} \partial^{\alpha} h_{\mu \nu} \partial_{\alpha} h^{\mu \nu}+\partial_{\mu} h^{\mu \nu} \partial_{\nu} h^{\alpha} \alpha-\frac{1}{2} \partial_{\alpha} h^{\mu}{ }_{\mu} \partial^{\alpha} h^{\nu}{ }_{\nu}\right]
$$

After fixing the gauge, and canonically quantising in radial co-ordinates [1] evaluated the entanglement entropy of a spherical entangling surface. The difficulty in using the linearised action of gravitons on $S^{1} \times A d S_{3}$ to evaluate the entanglement entropy are the following:

- The action (4.1) is classically not conformal invariant so it is not clear that it is related to the theory of the spin-2 field on $S^{1} \times A d S_{3}$. 
- $S^{1} \times A d S_{3}$ is not a solution to the Einstein equation with a given cosmological constant. Therefore one would not obtain a quadratic action by considering spin-2 fluctuations about the backgound $S^{1} \times A d S_{3}$. Indeed one has to set the fluctuations along $S^{1}$ to be zero by hand if the cosmological constant is chosen so that the spatial components of the Einstein equations are satisfied.

Instead we consider the action in (4.1) on $S^{1} \times R^{3}$, fix gauge and obtain the partition function. This is done in appendix B We are led to evaluate the following gauge invariant partition function

$$
\mathcal{Z}=\int \mathcal{D} h_{i j}^{\perp} \exp \left[-\frac{1}{2} \int d^{4} x h^{\perp i j}\left(-\partial_{\tau}^{2}-\partial^{k} \partial_{k}\right) h_{i j}^{\perp}\right] .
$$

Here the derivative in the temporal direction picks out the Kaluza-Klein mass of $h_{i j}^{\perp}$ which is a transverse traceless spin-2 field on $R^{3}$ satisfying

$$
\partial^{i} h_{i j}^{\perp}=0, \quad h_{i}^{i}=0 .
$$

We now turn on the curvature in $R^{3}$, this will introduce curvature couplings. The principle we use to introduce the curvature couplings is the following. In 3 dimensions we can write the flat space Laplacian using the identity

$$
\begin{aligned}
h_{k}^{\perp}{ }^{n} \epsilon_{m}^{k l} \epsilon^{m i j} \partial_{l} \partial_{i} h_{j n}^{\perp} & =h_{k}^{\perp n}\left(\delta^{k i} \delta^{l j}-\delta^{k j} \delta^{l i}\right) \partial_{l} \partial_{i} h_{j n}^{\perp}, \\
& =-h^{k n \perp}\left(\partial^{i} \partial_{i}\right) h_{k n}^{\perp} .
\end{aligned}
$$

Note that in arriving at the identity, the traceless condition in (4.3) has been used. Therefore on $R^{3}$ we can re-write the flat space Laplacian by the square of the operator $\epsilon^{m i j} \partial_{i}$ Thus the partition function is rewritten as

$$
\mathcal{Z}=\int \mathcal{D} h_{i j}^{\perp} \exp \left[-\frac{1}{2} \int d^{4} x\left(-h^{\perp i j} \partial_{\tau}^{2} h_{i j}^{\perp}+h_{k}^{\perp}{ }^{n} \epsilon_{m}^{k l} \epsilon^{m i j} \partial_{l} \partial_{i} h_{j n}^{\perp}\right)\right] .
$$

To introduce curvature couplings we now promote the ordinary derivatives to covariant derivatives on $A d S_{3}$, this results in the

$$
\begin{aligned}
h_{k}^{\perp} \epsilon_{m}^{n} \epsilon^{m i j} \nabla_{l} \nabla_{i} h_{j n}^{\perp} & =h_{k}^{\perp}{ }^{n}\left(g^{k i} g^{l j}-g^{k j} g^{l i}\right) \nabla_{l} \nabla_{i} h_{j n}^{\perp} \\
& =h_{k}^{\perp n} g^{k i} g^{l j} \nabla_{l} \nabla_{i} h_{j n}^{\perp}-h^{\perp k n} \nabla^{2} h_{k n}^{\perp}, \\
& =h_{k}^{\perp}{ }^{n} g^{k i} g^{l j}\left(\nabla_{i} \nabla_{l} h_{j n}+R_{j m l i} h_{n}^{m \perp}+R_{n m l i} h_{j}{ }^{m}\right)-h^{\perp k n} \nabla^{2} h_{k n}^{\perp} \\
& =-2 h^{\perp k n} h_{k n}^{\perp}-h^{\perp k n} h_{k n}^{\perp}-h^{\perp k n} \nabla^{2} h_{k n}^{\perp} .
\end{aligned}
$$

Here the $\epsilon$ tensor in curved space along the 3 directions is defined by

$$
\epsilon^{i j k}=\frac{\tilde{\epsilon}^{i j k}}{\sqrt{g}}, \quad \tilde{\epsilon}^{123}=1
$$

To obtain the last line in (4.6) we have used $\nabla^{i} h_{i j}^{\perp}=0$, the traceless condition $h_{i}^{i \perp}=0$ and the Riemann curvature components of $A d S_{3}$ given in (2.31). It is important to note 
that this principle naturally determines a definite value of the curvature induced mass. ${ }^{9}$ Therefore the partition function of the spin- 2 field on $S_{1} \times A d S_{3}$ is given by

$$
\begin{aligned}
\mathcal{Z} & =\int \mathcal{D} h_{i j}^{\perp} \exp \left[-\frac{1}{2} \int \sqrt{g} d^{4} x h^{\perp i j}\left(-\partial_{\tau}^{2}-\nabla^{2}-3\right) h_{i j}^{\perp}\right] \\
& =\left[\frac{1}{\operatorname{det}\left(-\partial_{\tau}^{2}-\Delta_{(2)}-3\right)}\right]^{\frac{1}{2}} .
\end{aligned}
$$

The action consists of a Kaluza-Klein tower of traceless transverse symmetric spin-2 fields. From (C.5) we see that the Kaluza-Klein zero mode satisfies the Breitenlohner-Freedman bound for spin-2 field in $d=3$. In fact as will be subsequently seen, performing the same analysis with the traceless transverse spin- $s$ field, the mass for the Kaluza-Klein zero modes is given by $m_{s}^{2}=-(s+1)$ which satisfies the corresponding Breitenlohner-Freedman bound.

We can now proceed as before and evaluate the free energy of this theory. The eigenvalues of the spin-2 Laplacian on transverse, traceless spin-2 field on $A d S_{3}$ [20]

$$
\Delta_{(2)} \psi_{i j}^{\{\lambda, u\}}=-\left(\lambda^{2}+3\right) \psi_{i j}^{\{\lambda, u\}} .
$$

Therefore the free energy for the Kaluza-Klein tower of spin-2 Kaluza-Klein modes is given by

$$
\mathcal{F}_{q}=\frac{1}{2} \sum_{n} \int d \mu^{(2)} \log \left(\lambda^{2}+\frac{n^{2}}{q^{2}}\right)
$$

The $m_{s}^{2}=-3$ cancels the shift from the eigenvalue of the spin-2 Laplacian ensuring that the Kaluza-Klein zero mode has a gapless spectrum. The Plancherel measure is given by $[20,26]$

$$
d \mu^{(2)}=\frac{\operatorname{Vol}\left(A d S^{3}\right)}{\pi^{2}}\left(\lambda^{2}+4\right) d \lambda .
$$

Here again we have used the spin dependent factor $g(s)$ determined by [26]. Substituting the Plancherel measure in the expression for the entanglement entropy given in (2.9), we obtain

$$
\begin{aligned}
S_{q} & =\frac{1}{1-q} \int d \mu^{(2)}\left[q \log \left(1-e^{-2 \pi \lambda}\right)-\log \left(1-e^{2 \pi q \lambda}\right)\right], \\
& =\frac{(q+1)\left(121 q^{2}+1\right)}{180 q^{3}} \log (\epsilon) .
\end{aligned}
$$

We can now take the $q \rightarrow 1$ limit to obtain the entanglement entropy, which results in

$$
S_{1}=\frac{61}{45} \log (\epsilon)
$$

Note that this precisely coincides with the result obtained by [1]. The method relied on canonical quantization of the graviton in spherical coordinates. In [7] it was noted that

\footnotetext{
${ }^{9}$ We will subsequently see that the introducing the curvature couplings using this principle results in a scale invariant partition function. The intuition for introducing curvature couplings arises from the fact that higher spin equations of motion in 3 dimensions can be written as square of the operator involving $\epsilon^{m i j} \nabla_{i}[35,36]$.
} 
the above result can also be obtained by considering the thermodynamics in de-Sitter space which is conformally related to that of hyperbolic cylinder. This result obtains the entanglement entropy by integrating the energy density in de-Sitter space. However this depended on a reasonable definition of energy density which did not have a first principle derivation [8].

We have shown the result follows from evaluating the partition function of a KaluzaKlein tower of traceless transverse spin-2 fields $S^{1} \times A d S_{3}$. The constant mode of the graviton on $S^{1}$ has the mass which saturates the Breitenlohner-Freedman bound. The mass in (4.8) was introduced using a definite principle for coupling the traceless transverse spin-2 fields to $A d S_{3}$. At present we do not have a first principle derivation that this theory is conformally related to that of the graviton in flat space. However, it is easy to see should the mass be any other mass other than $\left.m_{s}^{2}\right|_{s=2}=-3$, the partition function would not be just a function of the ratio of the length scales of $S^{1}$ and $A d S_{3}$. To see this we re-instate the radius of $A d S_{3}$ and also introduce an additional mass for the spin-2 field. Thus the starting point is given by

$$
\begin{aligned}
\mathcal{Z} & =\int \mathcal{D} h_{i j}^{\perp} \exp \left[-\frac{1}{2} \int \sqrt{g} d^{4} x h^{i j \perp}\left(-\partial_{\tau}^{2}-\nabla^{2}-3+m^{2}\right) h_{i j}^{\perp}\right] \\
& =\left[\frac{1}{\operatorname{det}\left(-\partial_{\tau}^{2}-\Delta_{(2)}-3+m^{2}\right)}\right]^{\frac{1}{2}}
\end{aligned}
$$

Evaluating the partition function using the eigen functions on $A d S_{3}$, we obtain

$$
\begin{aligned}
\mathcal{F}_{q} & =\frac{1}{2} \sum_{n} \int d \mu^{(2)} \log \left(\frac{\lambda^{2}}{R^{2}}+m^{2}+\frac{n^{2}}{q}\right) \\
& =\int d \mu^{(2)}\left(\log \left(1-e^{-2 \pi q \sqrt{\frac{\lambda^{2}}{R^{2}}+m^{2}}}\right)+\pi q \sqrt{\frac{\lambda^{2}}{R^{2}}+m^{2}}\right) .
\end{aligned}
$$

We can measure the additional mass $m^{2}$ in terms of units of the radius of $A d S_{3}$ by introducing $\hat{m}^{2}=R^{2} m^{2}$. Then the partition function becomes

$$
\mathcal{F}_{q}=\int d \mu^{(2)}\left(\log \left(1-e^{-2 \pi \frac{q}{R} \sqrt{\lambda^{2}+\hat{m}^{2}}}\right)+\pi \frac{q}{R} \sqrt{\lambda^{2}+\hat{m}^{2}}\right) .
$$

Note the $\mu^{(2)}$ is given in (4.11). From (4.16) we see that the free energy of the theory is a function $\mathcal{F}_{q}(q / R, \hat{m})$. The free energy of a scale invariant theory on $S^{1} \times A d S_{3}$ should be a function only of the ratio of the radius of $S^{1}$ to $A d S_{3}$. From (4.16), we see that this property holds only if and only if $\hat{m}=0$. Thus we have shown that it is only when the mass saturates the Breitenlohner-Freedman bound, the theory is scale invariant.

Finally we can proceed to evaluate the dimension of the twist operator localized on the entangling surface using the expression in (2.11). This results in

$$
\begin{aligned}
h_{q} & =\frac{q}{3 \pi} \int_{0}^{\infty} d \lambda \lambda\left(\lambda^{2}+4\right)[\operatorname{coth}(\pi \lambda)-\operatorname{coth}(\pi q \lambda)], \\
& =\frac{\left(q^{2}-1\right)\left(41 q^{2}+1\right)}{360 \pi q^{3}} .
\end{aligned}
$$


Examining the expressions for the Rényi entropy and $h_{q}$ in (4.12) and (4.17) and their counterparts for the spin- 0 and spin- 1 discussed earlier, we observe that Rényi entropy and the conformal dimensions of the twist operator are certain integral transforms of the Plancherel measure of the corresponding field.

\subsection{Higher spin fields in $D=4$}

Just as in the case of the graviton we can begin with the action of higher spin fields in flat space, fix gauge and arrive at the following gauge invariant action partition function for traceless transverse spin- $s$ tensors on $S^{1} \times R^{3}$.

$$
\mathcal{Z}=\int \mathcal{D} h_{i j}^{\perp} \exp \left[-\frac{1}{2} \int d^{4} x h^{\perp i_{1} i_{2}, \cdots i_{s}}\left(-\partial_{\tau}^{2}-\partial^{k} \partial_{k}\right) h_{i_{1} i_{2}, \cdots i_{s}}^{\perp}\right] .
$$

where $h_{i_{1}}^{\perp}, \ldots i_{s}$ are totally symmetric tensors satisfying

$$
\partial^{i} h_{i, i_{2}, \cdots i_{s}}^{\perp}=0, \quad h_{i i_{2} \cdots i_{s}}^{\perp i}=0 .
$$

We now introduce curvature couplings through the same principle. We can re-write the Laplacian in (4.18) in terms of the $\epsilon$ tensor as

$$
\begin{aligned}
h_{k}^{\perp n_{2}, \cdots n_{s}} \epsilon_{m}^{k l} \epsilon^{m i j} \partial_{l} \partial_{i} h_{j n_{2}, \cdots n_{s}}^{\perp} & =h_{k}^{\perp n_{2}, \cdots n_{s}}\left(\delta^{k i} \delta^{l j}-\delta^{k j} \delta^{l i}\right) \partial_{l} \partial_{i} h_{j n_{2}, \cdots n_{s}}^{\perp} \\
& =-h^{\perp j_{1} j_{2}, \cdots j_{s}}\left(\partial^{i} \partial_{i}\right) h_{j_{1} j_{2}, \cdots j_{s}}
\end{aligned}
$$

In arriving at the identity, the traceless condition in (4.19) has been used. As before we promote the ordinary derivatives to covariant derivatives on $A d S_{3}$, this results in the

$$
\begin{aligned}
& h_{k}^{\perp} n_{2}, \cdots n_{s} \epsilon_{m}^{k l} \epsilon^{m i j} \nabla_{l} \nabla_{i} h_{j n_{2} \cdots n_{s}}^{\perp}, \\
& =h_{k}^{\perp n_{2} \cdots n_{s}}\left(g^{k i} g^{l j}-g^{k j} g^{l i}\right) \nabla_{l} \nabla_{i} h_{j n_{2} \cdots n_{s}}^{\perp}, \\
& =h_{k}^{n \perp} g^{k i} g^{l j} \nabla_{l} \nabla_{i} h_{j n}^{\perp}-h^{k n \perp} \nabla^{2} h_{k n}^{\perp}, \\
& =h_{k}^{\perp n_{2} \cdots n_{s}} g^{k i} g^{l j}\left(\nabla_{i} \nabla_{l} h_{j n_{2} \cdots n_{s}}^{\perp}+R_{j m l i} h_{n_{2} \cdots n_{s}}^{m}+R_{n_{2} m l i} h_{j}^{\perp}{ }_{n_{3} \cdots n_{s}}\right. \\
& \left.+R_{n_{3} m l i} h_{j n_{2}}^{\perp} m{ }_{n_{4} \cdots n_{s}}+\cdots\right) \\
& -h^{\perp i_{1} \cdots i_{s}} \nabla^{2} h_{i_{1} \cdots i_{s}}^{\perp}, \\
& =-2 h^{\perp i_{1} \cdots i_{s}} h_{i_{1} \cdots i_{s}}^{\perp}-(s-1) h^{\perp i_{1} \cdots i_{s}} h_{i_{1} \cdots i_{s}}^{\perp}-h^{\perp i_{1} \cdots i_{s}} \nabla^{2} h_{i_{1} \cdots i_{s}}^{\perp} .
\end{aligned}
$$

To obtain the last line we have used $\nabla^{j} h_{j i_{2} \cdots i_{s}}^{\perp}=0$ and the traceless condition $h_{j i_{2} \cdots i_{s}}^{\perp j}=0 .{ }^{10}$

The partition function for these tower of Kaluza-Klein traceless transverse spin- $s$ particles are given by

$$
\begin{aligned}
& \mathcal{Z}=\int \mathcal{D} h_{i j}^{\perp} \exp \left[-\frac{1}{2} \int \sqrt{g} d^{4} x h^{\perp i_{1} \cdots i_{s}}\left(-\partial_{\tau}^{2}-\nabla^{2}-(s+1)\right) h_{i j}^{\perp}\right] \\
& =\left[\frac{1}{\operatorname{det}\left(-\partial_{\tau}^{2}-\Delta_{(2)}-(s+1)\right)}\right]^{\frac{1}{2}} .
\end{aligned}
$$

\footnotetext{
${ }^{10}$ As discussed for the spin-2 field, introducing curvature couplings using this principle results in a scale invariant partition function also for the spin-s fields. In fact the action $S=\int d^{3} \sqrt{g} h^{\perp i_{1} \cdots i_{s}} \epsilon_{i_{1}}^{j k} \nabla_{j} h_{k i_{2} \cdots i_{s}}^{\perp}$ is invariant under the Weyl transformation $g_{i j} \rightarrow e^{2 \phi} g_{i j}$ and $h_{i_{1} \cdots i_{s}}^{\perp} \rightarrow e^{(s-1) \phi} h_{i_{1} \cdots i_{s}}^{\perp}$. The Chern-Simons action is a very special case of this action which is topological. This perhaps might be the reason that the curvature couplings induced using this principle result in a scale invariant partition function.
} 
Again from (C.5) we see that mass of the Kaluza-Klein zero mode on $S^{1}$ given by $m_{s}^{2}=$ $-(s+1)$ satisfies the Brietenlohner-Freedman bound of the spin- $s$ field in $d=3$. We can evaluate the free energy using eigenfunction of the spin- $s$ Laplacian whose eigenvalues are given in (C.9). This results in

$$
\begin{aligned}
\mathcal{F}_{q} & =\frac{1}{2} \sum_{n} \int d \mu^{(s)} \log \left(\lambda^{2}+\frac{n^{2}}{q^{2}}\right), \\
d \mu^{(s)} & =\frac{\operatorname{Vol}\left(A d S_{3}\right)}{\pi^{2}}\left(\lambda^{2}+s^{2}\right) d \lambda .
\end{aligned}
$$

Here we have taking the Plancherel measure from [20, 26]. Evaluating the Rényi entropy using (2.9) we obtain

$$
\begin{aligned}
S_{q} & =\frac{1}{1-q} \int d \mu^{(s)}\left[q \log \left(1-e^{-2 \pi \lambda}\right)-\log \left(1-e^{2 \pi q \lambda}\right)\right], \\
& =\frac{(q+1)\left[\left(30 s^{2}+1\right) q^{2}+1\right]}{180 q^{3}} \log \epsilon .
\end{aligned}
$$

The entanglement entropy is given by

$$
S_{1}=\frac{15 s^{2}+1}{45} \log \epsilon .
$$

This result agrees with the conjecture of [1] as well as the result evaluated by [7] using thermodynamic of de-Sitter space. In addition it shows that the entanglement entropy is an integral transform of the Plancherel measure. The conformal dimension of the twist operator localised on the entangling surface is given by

$$
\begin{aligned}
h_{q} & =\frac{q}{3 \pi} \int_{0}^{\infty} d \lambda \lambda\left(\lambda^{2}+s^{2}\right)[\operatorname{coth}(\pi \lambda)-\operatorname{coth}(\pi q \lambda)], \\
& =\frac{\left(q^{2}-1\right)\left(q^{2}\left(10 s^{2}+1\right)+1\right)}{360 \pi q^{3}} .
\end{aligned}
$$

\subsection{Consistency check}

Let us perform another consistency check on the result of the entanglement entropy derived form using the theory of the Kaluza-Klein tower of spin-s particles on $S^{1} \times A d S_{3}$. We know that for conformal invariant theories, the result from evaluating the entanglement entropy of the theory on branched spheres should coincide with that of hyperbolic cylinders modulo the edges modes. We have discussed earlier for the Maxwell theory, the hyperbolic cylinders misses out the contribution of the edge modes, this same is true for the conformal $D / 2-1$-form theory in $D$ dimensions [28]. Let us check if this fact is true for the theory of higher spin fields in $D=4$ dimensions. The coefficient of the logarithmic divergence of the partition function for spin- $s$ fields on the $q$ branched sphere in $D=4$ has been evaluated in equation (E.4) of [37]. This is given by ${ }^{11}$

$$
\begin{aligned}
F[q] & =-\log Z=\zeta(q, s), \\
& =-\frac{1}{180 q^{3}}+\frac{\left(1-s^{2}\right) s^{2}}{6 q^{2}}-\frac{\left(3-2 s^{2}\right) s^{2}}{6 q}-\frac{1}{60} q\left(30 s^{4}-20 s^{2}+1\right)+\frac{1}{6}\left(1-3 s^{2}\right) s^{2} .
\end{aligned}
$$

\footnotetext{
${ }^{11}$ We have used the fact that cutoff $\Lambda_{U V}$ in [37] is in momentum space and therefore related to a position space cut off $\epsilon \sim \frac{1}{\Lambda_{U V}}$. We thank Arkady Tseytlin for bringing this reference to our attention after the first version of our manuscript appeared on the arXiv.
} 
Using this we evaluate the logarithmic coefficient of the entanglement entropy as given by the partition function on the branched sphere.

$$
\begin{aligned}
S_{q}\left(S_{q}^{4}\right) & =\frac{q F[1]-F[q]}{1-q}, \\
& =\frac{q\left(q^{2}+30(q(2 q-1)+1) s^{4}+30(q(q+2)-1) s^{2}+q+1\right)+1}{180 q^{3}} . \\
S_{1}\left(S^{4}\right) & =\lim _{q \rightarrow 1} S_{q}\left(S_{q}^{4}\right), \\
& =\frac{1}{45}\left(15\left(s^{4}+s^{2}\right)+1\right) .
\end{aligned}
$$

Let us now evaluate the difference between the entanglement entropies evaluated by considering the branched sphere and the theory of the Kaluza-Klein tower of transverse spin- $s$ particles on $S^{1} \times A d S_{3}$ given in (4.25). The logarithmic coefficient of the difference is given by

$$
S_{1}\left(S^{4}\right)-S_{1}\left(A d S_{3} \times S^{1}\right)=\frac{s^{4}}{3} .
$$

This should coincide with the partition function of the edge modes. Indeed, we see that for the Maxwell case, $s=1$ it agrees with the edge mode partition function found in $[2,3]{ }^{12}$ Recently the contribution of the edge modes for arbitrary spin in $D=4$ was evaluated in [15]. From this work we see that logarithmic contribution to the partition function from the edge modes for spin $s$ fields in $D=4, d=3$ is obtained from

$$
\begin{aligned}
\log \mathcal{Z}_{\text {edge }} & =\int \frac{d t}{2 t} \frac{1+q}{1-q}\left(-\left[\hat{\chi}_{\text {edge }, s}\right]_{+}\right), \quad q=e^{-t}, \\
{\left[\hat{\chi}_{\text {edge }, s}\right]_{+} } & =\frac{1}{1-q}\left[\frac{1}{3} s(s+1)(2 s+1) q^{s}-\frac{1}{3}(s-1) s(2 s-1) q^{s+1}\right] .
\end{aligned}
$$

These are read out from equation (5.11) and the second line of equation ( 5.9) of [15]. To obtain the logarithmic coefficient we need to perform the Taylor series expansion of the integrand in $t$ and extract out the coefficient of $1 / t$, this results in

$$
S_{\text {edge }}=\frac{s^{4}}{3}
$$

The contribution of the edge modes in (4.32) precisely agrees with the difference in the entanglement entropies on the sphere and that given on $S^{1} \times A d S_{3}$ in (4.30). This agreement provides a strong consistency check that the theory of the tower of Kaluza-Klein traceless transverse spin- $s$ fields at the Brietenlohner-Freedman bound which includes the case of the graviton is related to the theory of higher spin fields in flat space, as well as the theory of spin- $s$ on the sphere.

Finally we would like to point out that the agreement of entanglement entropy evaluated from the theory of higher spin fields on $S^{1} \times A d S_{3}$ given in (4.25) with that evaluated by [1] should not be a surprise given the structure of the Plancherel measure of the higher spin fields on $A d S_{3}$. From (4.23) and (4.24), we see that spin dependence arises only from

\footnotetext{
${ }^{12}$ See equation (46) of [3].
} 
the Plancherel measure and it is quadratic in spin, thus the logarithmic coefficient of the entanglement entropy is a function of the form

$$
S_{1}\left(S^{1} \times A d S_{3}\right)=A+B s^{2} .
$$

The mode counting argument of Benedetti and Casini in [1] also show that the entanglement entropy is also of form

$$
S_{1}(\mathrm{BC})=A^{\prime}+B^{\prime} s^{2}
$$

For spins $s=0, s=1$, the theories are conformally invariant and their actions on conformally flat spaces are related. Now for $s=0$ and $s=1$, we know $S_{1}\left(S^{1} \times A d S_{3}\right)=S_{1}(\mathrm{BC})$, therefore the agreement for arbitrary spin- $s$ is not a surprise.

\subsection{Symmetric tensors in arbitrary $D$}

In this section for completeness and future reference, we evaluate the free energy of the Kaluza-Klein tower of symmetric traceless rank-s tensors on $A d S_{d}, D=d+1$ is even. The mass of these fields saturate the Breitenlohner-Freedman bound. We then use the expression (2.9) to evaluate the Rényi entropies and entanglement entropy. The result for all even $D$ dimensions is given by

$$
S_{q}=\frac{1}{1-q} \int d \mu^{(s)}\left[q \log \left(1-e^{-2 \pi \lambda}\right)-\log \left(1-e^{2 \pi q \lambda}\right)\right],
$$

where the Plancherel measure is given by $[20,26]$

$$
\begin{aligned}
\mu^{(s)}(\lambda) d \lambda & =\frac{\operatorname{Vol}\left(A d S_{d}\right) g(s)}{2^{d-1} \pi^{\frac{d}{2}} \Gamma\left(\frac{d}{2}\right)}\left[\lambda^{2}+\left(s+\frac{d-3}{2}\right)^{2}\right] \prod_{j=0}^{\frac{d-5}{2}}\left(j^{2}+\lambda^{2}\right) d \lambda \\
g(s) & =\frac{(2 s+d-3) \Gamma(s+d-3)}{\Gamma(d-2) s !} .
\end{aligned}
$$

The spin dependent factor $g(s)$ was determined in [26] using analytical continuation of the heat kernel on $S^{d}$. For $d=3, g(0)=1$ and $g(s)=2$ for all $s \geq 1$. As we have seen in the previous section that fact that this factor of 2 is important for the result of the entanglement entropy of gravitons to precisely agree with that obtained in [1]. For $d>3$, $g(s)$ depends non-trivially on spin. The regularized volume of $A d S_{d}$ is given in [16].

$$
\operatorname{Vol}\left(A d S_{d}\right)=\frac{2 \pi^{\frac{d-1}{2}}(-1)^{\frac{d+1}{2}}}{\Gamma\left(\frac{d+1}{2}\right)} \log (\epsilon) .
$$

Table 2. lists the coefficient of the logarithm for entanglement entropies of the spin- $s$ field for all even $D$ dimensions $4 \leq D \leq 14$. The last column of this table takes the $s \rightarrow 0$ limit. The values agrees with the result for the entanglement entropy of a conformally coupled scalar across spheres [17].

The Rényi entropies are listed in appendix E. Finally we can also evaluate the conformal dimensions of the twist operator localized on the entangling surface using (2.11). This leads 


\begin{tabular}{|c|l|c|}
\hline$D$ & Coefficient of $\log (\epsilon)$ & $s \rightarrow 0$ \\
\hline 4 & $\frac{1+15 s^{2}}{45}$ & $\frac{1}{90}^{(*)}$ \\
6 & $-\frac{(s+1)^{2}\left(7 s^{2}+14 s+10\right)}{7560 s !}$ & $-\frac{1}{756}$ \\
8 & $\frac{(s+1)(s+2)^{2}(s+3)\left(5 s^{2}+20 s+23\right)}{1360800}$ & $\frac{23}{113400}$ \\
10 & $-\frac{(s+3)\left(759 s^{2}+4554 s+7364\right)(s+5) !}{75442752000 s !}$ & $-\frac{263}{7484400}$ \\
12 & $\frac{(s+4)\left(23933 s^{2}+191464 s+401361\right)(s+7) !}{1235752277760000 s !}$ & $\frac{133787}{20432412000}$ \\
14 & $-\frac{(s+5)\left(133787 s^{2}+1337870 s+3454198\right)(s+9) !}{4893579019929600000 s !}$ & $-\frac{157009}{122594472000}$ \\
\hline
\end{tabular}

${ }^{(*)}$ : for $D=4$ we have taken the limit $s \rightarrow 0$ first and then taken $D \rightarrow 4$. The order of limits matters for this case due to the nature of the function $g(s)$ in (4.35).

Table 2. Entanglement entropies of spin- $s$ fields in $D$ dimensions.

to the following integral

$$
h_{q}=\frac{q g(s)}{2^{d-1} \pi^{\frac{d}{2}-1} d \Gamma\left(\frac{d}{2}\right)} \int_{0}^{\infty} d \lambda \lambda\left[\lambda^{2}+\left(s+\frac{d-3}{2}\right)^{2}\right] \prod_{j=0}^{\frac{d-5}{2}}\left(j^{2}+\lambda^{2}\right)[\operatorname{coth}(\pi \lambda)-\operatorname{coth}(\pi q \lambda)] .
$$

These polynomials in $q$ for various dimensions are listed in the appendix E

\section{Conclusions}

We have evaluated the partition function of the $\mathrm{U}(1)$ in $D=4$ and the 2-form theory in $D=6$ starting from a gauge invariant action on the hyperbolic cylinder. After fixing gauge by adapting the functional methods of [10-12], we demonstrate that for these cases, the partition function reduces to a Kaluza-Klein tower of states with the corresponding spin in AdS space. For the conformal U(1) theory and the 2-form theory, the mass of the constant mode along $S^{1}$ satisfies the Brietenlohner-Freedman bound. We have verified that the entanglement entropy as well conformal dimensions of the co-dimension 2 twist operator of the $\mathrm{U}(1)$ and the 2-form theory satisfies known consistency checks.

The spin-2 theory in $D=4$ is not conformally invariant, we begin with the gauge invariant partition function of gravitons on $S^{1} \times R^{3}$ and using a principle to introduce curvature couplings, we obtain the partition function of the Kaluza-Klein tower of traceless transverse spin-2 fields. Again the mass of the constant mode along $S^{1}$ saturates the Brietenlohner-Freedman bound. This partition function is show to be scale invariant. The logarithmic coefficient of the entanglement entropy evaluated from this for the spin-2 theory agrees with that obtained by [1]. We repeat this analysis for higher spin fields in $D=4$ and show that the result coincides with the conjecture in [1]. Since the theory of gravitons in $D=4$ is not conformal invariant, as a consistency check on the application of the hyperbolic cylinder approach to the case of gravitons we demonstrate that the edge modes 
for arbitrary spin fields obtained from this approach precisely coincides with that recently obtained in [15].

In all the three cases, both the entanglement entropy and the weight of the twist operator are obtained by simple integral transforms of the Plancherel measure of the particle with the corresponding spin in AdS space. This resulted from the fact that the partition functions consisted of a tower of Kaluza-Klein fields on the hyperbolic cylinder with corresponding spin whose constant modes on $S^{1}$ satisfied the Brietenlohner-Freedman bound. It will be interesting to generalise this discussion to higher spin fermions and observe if the same conclusions hold. One can adapt the functional methods applied for the gravitino in [11].

The logarithmic coefficient of the entanglement entropy for the $\mathrm{U}(1)$ theory and the 2-form theory obtained by evaluating the partition function on the hyperbolic cylinder do not agree with the expected trace anomaly coefficient. The shift to restore this coefficient to the anomaly is attributed to the edge modes or the extended Hilbert space. Recently, in [25] it was shown that indeed, for the free Maxwell field without the presence of charged states, it is possible for this coefficient to be different from that of the trace anomaly. It was further shown that coupling with heavy charges changes this coefficient to that of the trace anomaly. It will be interesting to repeat this exercise for graviton or the 2-form theory to understand this phenomenon further.

\section{A Change in measures}

In this appendix we evaluate the change in the functional integration measure for the various change of variables used in performing the path integrals in the main text. We follow the methods developed in [10].

Maxwell field in $\boldsymbol{D}=4$. From the canonical measure $\mathcal{D} A_{\mu}$ we have the result

$$
\int \mathcal{D} A_{\mu} e^{-\int d^{4} x \sqrt{g} A_{\mu} A^{\mu}}=1
$$

Let us change variables to

$$
\left\{A_{\mu}\right\} \rightarrow\left\{A_{\tau}, A_{i}^{\perp}, \phi\right\}
$$

where $i$ refers to the $A d S_{3}$ directions and $\nabla^{i} A_{i}^{\perp}=0$. We can write the term in the exponent as

$$
\int d^{4} x \sqrt{g} A_{\mu} A^{\mu}=\int d^{4} x \sqrt{g}\left(A_{\tau}^{2}+A_{i}^{\perp} A^{\perp i}+\phi\left(-\Delta_{(0)}\right) \phi\right) .
$$

We have used integration by parts and the transversal property of $A_{i}^{\perp}$ to arrive at the above equation. $\Delta_{(0)}$ is the scalar Laplacian on $A d S_{3}$. Let determinant of the Jacobian involved in the change of measure be $J$. Then from (A.1) we obtain

$$
\int \mathcal{D} A_{\tau} \mathcal{D} A_{i}^{\perp} \mathcal{D} \phi J e^{-\int d^{4} x \sqrt{g}\left(A_{\tau}^{2}+A_{i}^{\perp} A^{\perp i}+\phi\left(-\Delta_{(0)}\right) \phi\right)}=1 .
$$

Now performing all the functional integrals, we obtain

$$
J\left[\operatorname{det}\left(-\Delta_{(0)}\right)\right]^{-\frac{1}{2}}=1 .
$$


Therefore the change in measure for the transformation given in (A.2), we obtain

$$
\mathcal{D} A_{\mu}=\mathcal{D} A_{\tau} \mathcal{D} A_{i}^{\perp} \mathcal{D} \phi \times J, \quad J=\left[\operatorname{det}\left(-\Delta_{(0)}\right)\right]^{\frac{1}{2}} .
$$

Graviton in $\boldsymbol{D}=4: \boldsymbol{S}^{\mathbf{1}} \times \boldsymbol{R}^{\mathbf{3}}$. The fields for the graviton we have are $h_{\tau \tau}, h_{\tau i}$ and $h_{i j}$. We start with the canonical definition of he measure given by the integral

$$
\int \mathcal{D} h_{\tau \tau} \mathcal{D} h_{\tau i} \mathcal{D} h_{i j} e^{-\int d^{4} x\left(h_{\tau \tau}^{2}+2 h_{\tau i} h^{\tau i}+h_{i j} h^{i j}\right)}=1 .
$$

The coordinates $i, j$ refer to $R^{3}$ directions. We decompose $h_{i j}$ into its traces and traceless components by

$$
h_{i j}=\bar{h}_{i j}+\frac{1}{3} \delta_{i j} h
$$

The traceless component $\bar{h}_{i j}$ can be further decomposed as

$$
\begin{aligned}
\bar{h}_{i j} & =h_{i j}^{\perp}+\partial_{i} \zeta_{j}^{\perp}+\partial_{j} \zeta_{i}^{\perp}+\partial_{i} \partial_{j} \sigma-\frac{1}{3} \delta_{i j} \square \sigma, \\
\partial^{i} h_{i j}^{\perp} & =0, \quad \partial^{i} \zeta_{i}^{\perp}=0 .
\end{aligned}
$$

With these definitions we have

$$
\begin{aligned}
\int d^{4} x h_{i j} h^{i j}=\int d^{4} x\left[h_{i j}^{\perp} h^{\perp i j}\right. & +\left(\partial_{i} \zeta_{j}^{\perp}+\partial_{j} \zeta_{i}^{\perp}\right)^{2} \\
& \left.+\left(\partial_{i} \partial_{j} \sigma-\frac{1}{3} \delta_{i j} \square \sigma\right)^{2}+\frac{1}{3} h^{2}\right] .
\end{aligned}
$$

The cross terms cancel due to integration by parts due to the transverse gauge condition as well as the traceless condition. Substituting this, we can further simplify the terms by integration by parts and obtain

$$
\begin{aligned}
\int d^{4} x \sqrt{g} h_{i j} h^{i j}=\int d^{4} x \sqrt{g}\left[h_{i j}^{\perp} h^{\perp i j}+2 \zeta_{i}^{\perp}\left(-\Delta_{(1)}\right)\right. & \zeta^{i \perp} \\
& \left.+\frac{2}{3} \sigma \Delta_{(0)} \Delta_{(0)} \sigma+\frac{1}{3} h^{2}\right] .
\end{aligned}
$$

Here $\Delta_{(1)}$ spin-1 Laplacian for transverse fields on $R^{3}$. We treat $h_{\tau i}, h_{i j}^{\perp}, \zeta_{i}{ }^{\perp}, \sigma$ and $h$ as independent set of variables and substitute (A.11) in (A.7). Performing the resultant integrals just as in the case of the $\mathrm{U}(1)$ field we obtain the change in measure as

$$
\mathcal{D} h_{i j}=\mathcal{D} h_{i j}^{\perp} \mathcal{D} \zeta_{i}^{\perp} \mathcal{D} \sigma \mathcal{D} h J, \quad J=\left[\operatorname{det}\left(\left(-\Delta_{(1)}\right)\left(-\Delta_{(0)}\right)^{2}\right)\right]^{\frac{1}{2}} .
$$

Similarly, following the same steps for the Maxwell field decomposing $h_{\tau i}$ into its transverse and longitudinal component along $A d S_{3}$ results in the following change in measure

$$
\mathcal{D} h_{\tau i}=\mathcal{D} h_{\tau i}^{\perp} \mathcal{D} \phi J, \quad J=\left[\operatorname{det}\left(-\Delta_{(0)}\right)\right]^{\frac{1}{2}} .
$$


2-form in $\boldsymbol{D}=\mathbf{6}$. Again we start with the canonical normalization

$$
\int \mathcal{D} A_{\mu \nu} e^{-\int d^{6} x \sqrt{g}\left(A_{\mu \nu} A^{\mu \nu}\right)}=1
$$

We decompose $A_{\mu \nu}$ in the components of $A_{\tau i}$ and $A_{i j}$. where $i, j$ denotes the components along $A d S_{5}$ directions. We can further decompose $A_{i j}$ in the following way

$$
A_{i j}=A_{i j}^{\perp}+\nabla_{i} \chi_{j}^{\perp}-\nabla_{j} \chi_{i}^{\perp}, \quad \nabla_{i} A^{\perp i j}=0, \quad \nabla_{i} \chi^{\perp i}=0 .
$$

Note that the longitudinal component of $\chi^{i}$ does not occur in this decomposition. Now

$$
\int d^{6} x \sqrt{g}\left(A_{\mu \nu} A^{\mu \nu}\right)=\int d^{6} x \sqrt{g}\left[2\left(A_{\tau i}\right)^{2}+\left(A_{i j}^{\perp}\right)^{2}+2 \chi_{i}^{\perp}\left(-\Delta_{(1)}+4\right) \chi^{\perp i}\right] .
$$

where $\Delta_{(1)}$ is the spin-1 laplacian on $A d S_{5}$. Substituting the expansion in (A.16) into (A.14) and re-writing the integration interms of $A_{i j}^{\perp}, \chi_{i}^{\perp}$ we obtain the following equation for the change in measures

$$
\mathcal{D} A_{i j}=\mathcal{D} A_{i j}^{\perp} \mathcal{D} \chi_{i}^{\perp} J, \quad J=\left[\operatorname{det}\left(-\Delta_{(1)}+4\right)\right]^{\frac{1}{2}} .
$$

Similar to the $\mathrm{U}(1)$ case, one can decompose the component $A_{\tau i}$ in terms of its transverse and longitudinal component along $A d S_{3}$. The change in measure for this decomposition is given by

$$
\mathcal{D} A_{\tau i}=\mathcal{D} A_{\tau i}^{\perp} \mathcal{D} \chi_{\tau} J, \quad J=\left[\operatorname{det}\left(-\Delta_{(0)}\right)\right]^{\frac{1}{2}} .
$$

2-form gauge group: non-zero modes. Let us also evaluate the change in measure for the gauge group integrations. We do this for the non-zero Kaluza-Klein modes first. The reason we need these change of measures is because while gauge fixing, one divides out with the integrations over the measure $\mathcal{D} \epsilon_{\mu}$. However the gauge slice we choose is over transformations which involve the transverse $\epsilon_{i}^{\perp}$, and longitudinal component $\epsilon$, on $A d S_{5}$ We first normalise the gauge parameters $\epsilon_{\mu}$ as

$$
\int \mathcal{D} \epsilon_{\mu} e^{-\int d^{6} x \sqrt{g} \epsilon_{\mu} \epsilon^{\mu}}=1 .
$$

In the gauge transformation for the 2 -form (3.5), note that the transverse component $\epsilon_{\mu}^{\perp}$ over $S^{1} \times A d S_{5}$ occurs. Therefore we first make a change of variables by the transverse and longitudinal components of the gauge parameter on the entire $S^{1} \times A d S_{5}$ space. For this we write

$$
\epsilon_{\mu}=\epsilon_{\mu}^{\perp}+\nabla_{\mu} \tilde{\epsilon}, \quad \mu \in\{\tau, i\}
$$

Then we write (A.19) as

$$
\begin{aligned}
\int \mathcal{D} \epsilon_{\mu} e^{-\int d^{6} x \sqrt{g} \epsilon_{\mu} \epsilon^{\mu}} & =\int \mathcal{D} \epsilon_{\mu}^{\perp} \mathcal{D} \tilde{\epsilon} J_{1} e^{-\int d^{6} x \sqrt{g}\left[\epsilon_{\mu}^{\perp} \epsilon^{\perp \mu}+\tilde{\epsilon}\left(-\partial_{\tau}^{2}-\Delta_{(0)}\right) \tilde{\epsilon}\right]} \\
& =\int \mathcal{D} \epsilon_{\mu}^{\perp} J_{1}\left[\operatorname{det}\left(-\partial_{\tau}^{2}-\Delta_{(0)}\right)\right]^{-\frac{1}{2}} e^{-\int d^{6} x \sqrt{g} \epsilon_{\mu}^{\perp} \epsilon^{\perp \mu}}
\end{aligned}
$$

We can now further write $\epsilon_{\mu}^{\perp}$ in terms of $\epsilon$ and $\epsilon_{i}^{\perp}$

$$
\left\{\epsilon_{\mu}^{\perp}\right\} \rightarrow\left\{\epsilon, \epsilon_{i}^{\perp}\right\}, \quad \nabla^{i} \epsilon_{i}^{\perp}=0 .
$$


Note that here we need to eliminate $\epsilon_{\tau}$ in terms of $\epsilon$ using equation (3.11). Proceeding we write the integral as

$$
\begin{aligned}
\int d^{6} x \sqrt{g}\left(\epsilon_{\mu}^{\perp} \epsilon^{\perp \mu}\right) & =\int d^{6} x \sqrt{g}\left[\epsilon\left(\frac{-\Delta_{(0)}^{2}}{\partial_{\tau}^{2}}\right) \epsilon+\epsilon_{i}^{\perp} \epsilon^{\perp i}+\epsilon\left(-\Delta_{(0)}\right) \epsilon\right] \\
& =\int d^{6} x \sqrt{g}\left[\epsilon_{i}^{\perp} \epsilon^{\perp i}+\epsilon\left(\frac{1}{\partial_{\tau}^{2}}\left[-\partial_{\tau}^{2}-\Delta_{(0)}\right]\left(-\Delta_{(0)}\right)\right) \epsilon\right]
\end{aligned}
$$

To obtain the first line we have eliminated $\epsilon_{\tau}$ using (3.11). Now substituting the above equation in the exponential of (A.21), we obtain

$$
\begin{aligned}
& \int \mathcal{D} \epsilon_{\mu}^{\perp} J_{1}\left[\operatorname{det}\left(-\partial_{\tau}^{2}-\Delta_{(0)}\right)\right]^{-\frac{1}{2}} e^{-\int d^{6} x \sqrt{g} \epsilon_{\mu}^{\perp} \epsilon^{\perp \mu}} \\
& \quad=\int \mathcal{D} \epsilon_{i}^{\perp} \mathcal{D} \epsilon J_{1} J_{2}\left[\operatorname{det}\left(-\partial_{\tau}^{2}-\Delta_{(0)}\right)\right]^{-\frac{1}{2}} e^{-\int d^{6} x \sqrt{g}\left[\epsilon_{i}^{\perp} \epsilon^{\perp i}+\epsilon\left(\frac{1}{\partial_{\tau}^{2}}\left(-\partial_{\tau}^{2}-\Delta_{(0)}\right)\left(-\Delta_{(0)}\right)\right) \epsilon\right]}, \\
& \quad=J_{1} J_{2}\left[\operatorname{det}\left(\left(\frac{1}{\partial_{\tau}}\right)\left(-\partial_{\tau}^{2}-\Delta_{(0)}\right)\right)\right]^{-1}\left[\operatorname{det}\left(-\Delta_{(0)}\right)\right]^{-\frac{1}{2}}
\end{aligned}
$$

Using (A.19) and (A.21) and (A.24), we obtain the following change in measure

$$
\begin{aligned}
\mathcal{D} \epsilon_{\mu} & =\mathcal{D} \epsilon_{i}^{\perp} \mathcal{D} \epsilon \mathcal{D} \tilde{\epsilon} J \\
J & =J_{1} J_{2}=\left[\operatorname{det}\left(\left(\frac{1}{\partial_{\tau}}\right)\left(-\partial_{\tau}^{2}-\Delta_{(0)}\right)\right)\right]\left[\operatorname{det}\left(-\Delta_{(0)}\right)\right]^{\frac{1}{2}} .
\end{aligned}
$$

2-form gauge group: zero modes. Now let us find the change in the measure of the gauge parameter for the zero modes. Again we begin with the normalization in (A.19). For the zero modes The constraint $\nabla^{\mu} \epsilon_{\mu}=0$ reduces to the constraint $\nabla^{i} \epsilon_{i}=0$, so we write $\epsilon_{i}=\epsilon_{i}^{\perp}+\nabla_{i} \epsilon$. Then

$$
\int d^{6} x \sqrt{g} \epsilon_{\mu} \epsilon^{\mu}=\int d^{6} x \sqrt{g}\left(\epsilon_{\tau}^{2}+\epsilon_{i}^{\perp} \epsilon^{\perp i}+\epsilon\left(-\Delta_{0}\right) \epsilon\right) .
$$

We substitute this into (A.19) and obtain

$$
\begin{aligned}
\int \mathcal{D} \epsilon_{\mu} e^{-\int d^{6} x \sqrt{g} \epsilon_{\mu} \epsilon^{\mu}} & =\int \mathcal{D} \epsilon_{\tau} \mathcal{D} \epsilon_{i}^{\perp} \mathcal{D} \epsilon J e \int d^{6} x \sqrt{g}\left(\epsilon_{\tau}^{2}+\epsilon_{i}^{\perp} \epsilon^{\perp i}+\epsilon\left(-\Delta_{0}\right) \epsilon\right) \\
& =J\left[\operatorname{det}\left(-\Delta_{0}\right)\right]^{-\frac{1}{2}}=1
\end{aligned}
$$

Now comparing (A.27) and (A.19) we obtain

$$
\mathcal{D} \epsilon_{\mu}=\mathcal{D} \epsilon_{\tau} \mathcal{D} \epsilon_{i}^{\perp} \mathcal{D} \epsilon J \quad J=\left[\operatorname{det}\left(-\Delta_{0}\right)\right]^{\frac{1}{2}} .
$$

\section{B Partition function of the graviton: $S^{1} \times R^{3}$}

Consider the action of the graviton in flat space. This is given by

$$
S=\int d^{4} x\left[-\partial_{\mu} h^{\mu \nu} \partial_{\alpha} h_{\nu}^{\alpha}+\frac{1}{2} \partial^{\alpha} h_{\mu \nu} \partial_{\alpha} h^{\mu \nu}+\partial_{\mu} h^{\mu \nu} \partial_{\nu} h^{\alpha} \alpha-\frac{1}{2} \partial_{\alpha} h^{\mu}{ }_{\mu} \partial^{\alpha} h^{\nu}{ }_{\nu}\right] .
$$


To evaluate the partition function on $S^{1} \times R^{3}$ we gauge fix as done for the Maxwell field and the 2 -form in $D=6$. We first write the action in terms of $h_{\tau \tau}, h_{\tau i}, h_{i j}$. This is given by the following

$$
\begin{aligned}
S & =\int d^{4} x\left(\mathcal{L}_{1}+\mathcal{L}_{2}+\mathcal{L}_{3}\right), \\
\mathcal{L}_{1} & =h^{\tau j}\left(-\partial^{i} \partial_{i}\right) h_{\tau j}-\left(\partial_{i} h^{i \tau}\right)^{2}, \\
\mathcal{L}_{2} & =h \partial^{i} \partial_{i} h_{\tau \tau}+\partial^{i} h_{i j} \partial^{j} h_{\tau \tau}+2 \partial_{\tau} h_{\tau i}\left(\partial^{i} h-\partial_{j} h^{i j}\right), \\
\mathcal{L}_{3} & =\frac{1}{2} h^{i j}\left(-\partial_{\tau}^{2}-\partial^{k} \partial_{k}\right) h_{i j}-\frac{1}{2} h\left(-\partial_{\tau}^{2}-\partial^{k} \partial_{k}\right) h-\partial_{i} h^{i j} \partial^{k} h_{k j}+\partial_{i} h^{i j} \partial_{j} h .
\end{aligned}
$$

This action is invariant under

$$
\begin{aligned}
\delta h_{\tau \tau} & =2 \partial_{\tau} \epsilon_{\tau}, \\
\delta h_{\tau i} & =\partial_{\tau} \epsilon_{i}+\partial_{i} \epsilon_{\tau}, \\
\delta h_{i j} & =\partial_{i} \epsilon_{j}+\partial_{j} \epsilon_{i} .
\end{aligned}
$$

We decompose

$$
\begin{aligned}
h_{i j} & =\bar{h}_{i j}+\frac{1}{3} \delta_{i j} h, \\
\bar{h}_{i j} & =h_{i j}^{\perp}+\partial_{i} \zeta_{j}^{\perp}+\partial_{j} \zeta_{i}^{\perp}+\partial_{i} \partial_{j} \sigma-\frac{1}{3} \delta_{i j} \sigma, \\
\text { where } \quad \partial^{i} h_{i j}^{\perp} & =0, \quad \partial^{i} \zeta_{i}^{\perp}=0 .
\end{aligned}
$$

Under this change of variables the measure changes as (A.12)

$$
\begin{aligned}
\mathcal{D} h_{\tau \tau} \mathcal{D} h_{\tau i} \mathcal{D} h_{i j} & =\mathcal{D} h_{\tau \tau} \mathcal{D} h_{\tau i} \mathcal{D} h_{i j}^{\perp} \mathcal{D} \zeta_{i}^{\perp} \mathcal{D} h \mathcal{D} \sigma \times J \\
J & =\operatorname{det}\left[\left(-\Delta_{(1)}\right)\left(-\Delta_{(0)}\right)^{2}\right]^{\frac{1}{2}}
\end{aligned}
$$

Here we have used the subscript on the Laplacian's to denote that act on transverse vectors and scalars respectively. The measure can be split for the zero and the non-zero Kaluza Klein modes on $S^{1}$ as

$$
\begin{aligned}
\mathcal{D} h_{\tau \tau} \mathcal{D} h_{\tau i} \mathcal{D} h_{i j}= & {\left[\mathcal{D} h_{\tau \tau} \mathcal{D} h_{\tau i} \mathcal{D} h_{i j}^{\perp} \mathcal{D} \zeta_{i}^{\perp} \mathcal{D} h \mathcal{D} \sigma \times J\right]_{n \neq 0} } \\
& \times \mathcal{D} h_{\tau \tau} \mathcal{D} h_{\tau i} \mathcal{D} h_{i j}^{\perp} \mathcal{D} \zeta_{i}^{\perp} \mathcal{D}[\mathcal{D} h \mathcal{D} \sigma \times J]_{n=0} .
\end{aligned}
$$

Non-zero Kaluza-Klein modes. For the non-zero Kaluza-Klein modes, from the gauge transformation in (B.3) we can fix the gauge

$$
h_{\tau \tau}=0, \quad h_{\tau i}=0 .
$$

This gauge choice along with the Fadeev-Popov determinant is given by

$$
\mathcal{G}=\delta\left(h_{\tau \tau}\right)\left(h_{\tau i}\right)\left[\operatorname{det}\left(\partial_{\tau}\right)^{4}\right]
$$


Imposing the delta function constraints results in the following action

$$
\begin{aligned}
S & =\int d^{4} x\left(\mathcal{L}_{h^{\perp}}+\mathcal{L}_{\zeta^{\perp}}+\mathcal{L}_{3}^{\prime}\right)_{\sigma, h}, \\
\mathcal{L}_{h^{\perp}} & =\frac{1}{2} h^{i j \perp}\left(-\partial_{\tau}^{2}-\square\right) h_{i j}^{\perp}, \\
\mathcal{L}_{\zeta^{\perp}} & =\zeta^{i \perp}\left(-\partial_{\tau}^{2}\right)(-\square) \zeta_{i}^{\perp}, \\
\mathcal{L}_{\sigma, h} & =\frac{1}{9}\left[\sigma\left(-3 \partial_{\tau}^{2}+\square\right) \square^{2} \sigma-2 h \square^{2} \sigma+h\left(3 \partial_{\tau}^{2}+\square\right) h\right] .
\end{aligned}
$$

It is convenient to write the $\sigma, h$ part of the action as a matrix.

$$
\mathcal{L}_{\sigma, h}=\frac{1}{9}(\sigma h)\left(\begin{array}{cc}
\left(-3 \partial_{\tau}^{2}+\square\right) \square^{2} & -\square^{2} \\
-\square^{2} & \left(3 \partial_{\tau}^{2}+\square\right)
\end{array}\right)\left(\begin{array}{l}
\sigma \\
h
\end{array}\right) .
$$

Integrating out $h_{i j}^{\perp}, \zeta_{i}^{\perp}, \sigma, h$ we obtain

$$
\mathcal{Z}_{h_{i j}^{\perp}, \zeta_{i}^{\perp}, \sigma, h}=\left[\operatorname{det}\left(-\partial_{\tau}^{2}-\Delta_{(2)}\right)\left(-\Delta_{(1)}\right)\left(-\Delta_{(0)}\right)^{2}\left(\partial_{\tau}^{8}\right)\right]^{-\frac{1}{2}}
$$

Here we have used the fact that $\zeta_{i}^{\perp}$ has 2 independent degrees of freedom, therefore $\operatorname{det}\left(\partial_{\tau}^{2}\left(-\Delta_{(1)}\right)\right)=\left(\operatorname{det}\left(\partial_{\tau}\right)\right)^{4} \operatorname{det}\left(-\Delta_{(1)}\right) .{ }^{13}$ Now combining (B.11), the Jacobian in (B.5) and the Fadeev-Popov determinant (B.11) we obtain

$$
\mathcal{Z}_{n \neq 0}=\left[\operatorname{det}\left(-\partial_{\tau}^{2}-\Delta_{(2)}\right)\right]^{-\frac{1}{2}} .
$$

Zero modes. For the Kaluza-Klein zero modes, we see that $h_{\tau \tau}$ is gauge invariant, and we have

$$
\begin{aligned}
\delta h_{\tau \tau} & =0, \quad \delta h_{\tau i}=\partial_{i} \epsilon_{\tau}, \\
\delta h_{i j} & =\partial_{i} \epsilon_{j}+\partial_{j} \epsilon_{i} .
\end{aligned}
$$

Now we decompose the fields as

$$
\begin{aligned}
& h_{\tau i}=h_{\tau i}^{\perp}+\partial_{i} \phi, \quad \partial^{i} h_{\tau i}^{\perp}=0, \\
& h_{i j}=\bar{h}_{i j}+\frac{1}{3} \delta_{i j} h, \\
& \bar{h}_{i j}=h_{i j}^{\perp}+\partial_{i} \zeta_{j}^{\perp}+\partial_{j} \zeta_{i}^{\perp}+\partial_{i} \partial_{j} \sigma-\frac{1}{3} \delta_{i j} \sigma,
\end{aligned}
$$

where $\quad \partial^{i} h_{i j}^{\perp}=0, \quad \partial^{i} \zeta_{i}^{\perp}=0$.

This change of variable involves the Jacobian (A.12), (A.13)

$$
\begin{aligned}
\mathcal{D} h_{\tau \tau} \mathcal{D} h_{\tau i} \mathcal{D} h_{i j} & =\mathcal{D} h_{\tau \tau} \mathcal{D} h_{\tau i}^{\perp} \mathcal{D} \phi \mathcal{D} h_{i j}^{\perp} \mathcal{D} \zeta_{i}^{\perp} \mathcal{D} \sigma \mathcal{D} h \times J^{\prime} \\
J^{\prime} & =\operatorname{det}\left[\left(-\Delta_{(1)}\right)\left(-\Delta_{(0)}\right)^{3}\right]^{\frac{1}{2}}
\end{aligned}
$$

\footnotetext{
${ }^{13}$ The easiest way to see this is to work in momentum space and use the projector $\delta_{i j}-\frac{k_{i} k_{i}}{k^{2}}$ to ensure $\partial \zeta_{i}^{\perp}=0$.
} 
Decomposing the gauge parameter we have

$$
\epsilon_{i}=\epsilon_{i}^{\perp}+\frac{1}{2} \partial_{i} \epsilon .
$$

In terms of the components, the gauge transformation can be written as

$$
\delta h_{i j}^{\perp}=0, \quad \delta h_{\tau i}^{\perp}=0, \quad \delta \phi=\epsilon_{\tau}, \quad \delta \zeta_{i}^{\perp}=\epsilon_{i}^{\perp}, \quad \delta \sigma=\epsilon, \quad \delta h=\square \epsilon
$$

The action in terms of the components becomes

$$
\begin{aligned}
S & =\int d^{4} x\left(\mathcal{L}_{h_{\tau \tau}}+\mathcal{L}_{h_{\tau i}^{\perp}}+\mathcal{L}_{h_{i j}^{\perp}}+\mathcal{L}_{\sigma, h}\right), \\
\mathcal{L}_{h_{\tau \tau}} & =\frac{2}{3}(h-\square \sigma) \square h_{\tau \tau}, \\
\mathcal{L}_{h_{\tau i}^{\perp}} & =h^{\tau i \perp}(-\square) h_{\tau i}^{\perp}, \\
\mathcal{L}_{h_{i j}^{\perp}} & =\frac{1}{2} h^{i j \perp}(-\square) h_{i j}^{\perp}, \\
\mathcal{L}_{\sigma, h} & =\frac{1}{9}\left(\begin{array}{cc}
\sigma & h
\end{array}\right)\left(\begin{array}{cc}
\square^{3} & -\square^{2} \\
-\square^{2} & \square
\end{array}\right)\left(\begin{array}{l}
\sigma \\
h
\end{array}\right) .
\end{aligned}
$$

Note the absence of $\phi, \zeta_{i}^{\perp}$ in the action due to gauge invariance. We fix gauge by introducing

$$
\mathcal{G}=\delta(\phi) \delta\left(\zeta_{i}^{\perp}\right) \delta(\sigma)
$$

It is clear from the gauge transformations given in (B.17), the Fadeev-Popov determinant is unity. Since our gauge parameters are $\epsilon_{i}^{\perp}, \epsilon$ we need the following change of variables

$$
\mathcal{D} \epsilon_{i}^{\perp} \mathcal{D} \epsilon=\mathcal{D} \epsilon_{i} \times J^{\prime \prime}, \quad J^{\prime \prime}=\left[\frac{1}{\Delta_{(0)}}\right]^{\frac{1}{2}} .
$$

Now we can integrate over $\epsilon_{\tau}, \zeta_{i}^{\perp}, \sigma$, which implements the delta function. After which we can integrate over $h_{\tau, i}^{\perp}, h_{i j}^{\perp}, h$ and finally $h_{\tau \tau}$. This results in

$$
\mathcal{Z}_{h_{i j}^{\perp}, h_{\tau i}^{\perp}, h, \sigma, \zeta_{i}^{\perp}, h_{\tau \tau}}=\left\{\operatorname{det}\left[\left(-\Delta_{(2)}\right)\left(-\Delta_{(1)}\right)\left(-\Delta_{(0)}\right)^{2}\right]\right\}^{\frac{1}{2}} .
$$

Now putting together the Jacobian from the change of measure in (B.15), and that for the gauge transformation in (B.20) along with the result of the integrations in (B.21) we obtain the final result for the zero modes partition function to be given by

$$
\mathcal{Z}_{n=0}=\left[\frac{1}{\operatorname{det}\left(-\Delta_{(2)}\right)}\right]^{\frac{1}{2}} .
$$

Combining the result in (B.22) and (B.12) we obtain the following partition function for the graviton on $S^{1} \times R^{3}$

$$
\mathcal{Z}=\left[\operatorname{det}\left(-\partial_{\tau}^{2}-\Delta_{(2)}\right)\right]^{-\frac{1}{2}}
$$

where $-\partial_{\tau}^{2} \rightarrow n^{2} / q^{2}$ for each Kaluza-Klein mode and $\Delta_{(2)}$ is the Laplacian on transverse symmetry traceless tensor on $R^{3}$. The partition function can also be written as

$$
\mathcal{Z}=\int \mathcal{D} h_{i j}^{\perp} \exp \left[-\frac{1}{2} \int d^{4} x h^{i j \perp}\left(-\partial_{\tau}^{2}-\partial^{k} \partial_{k}\right) h_{i j}^{\perp}\right] .
$$




\section{Brietenholer-Freedman bound for higher spins}

The equations of motions of massive symmetric traceless tensors in $A d S_{d}$ dimensions ${ }^{14}$ are given by $[38,39]$

$$
\begin{aligned}
\left(\nabla^{2}-m_{s}^{2}\right) \Phi_{\mu_{1} \mu_{1} \cdots \mu_{s}} & =0 \\
\nabla^{\mu} \Phi_{\mu \mu_{2} \cdots \mu_{s}} & =0 \\
g^{\mu \nu} \Phi_{\mu \nu \mu_{3} \cdots \mu_{s}} & =0 .
\end{aligned}
$$

Here $\Phi_{\mu_{1}, \mu_{2}, \mu_{s}}$ is a totally symmetric rank $s$ tensor. The mass $m_{s}^{2}$ is given by

$$
m_{s}^{2}=\left[s^{2}+s(d-6)+6-2 d\right]+m^{2},
$$

The term in the square brackets is the natural mass that exists due to the curvature of $A d S_{d}$. Note that it also exists when $m=0$, in fact 'massless' higher spin fields in $A d S_{d}$ have $m=0$. The putative dual operator corresponding to the higher spin field by the $A d S_{d} / C F T_{d-1}$ corresponds to an operator of spin $s$ with conformal dimension [38]

$$
\Delta=\frac{d-1}{2}+\sqrt{m^{2}+\left(s+\frac{d-5}{2}\right)^{2}} .
$$

For the reader who is familiar with this dictionary for scalars, note that taking $s=0$, the expression for the scaling dimension reduces to

$$
\Delta=\frac{d-1}{2}+\sqrt{m_{s=0}^{2}+\left(\frac{d-1}{2}\right)^{2}} .
$$

This is the standard formula for the conformal dimension of the operator dual to the minimally coupled scalar of mass $m_{s=0}^{2}$ in $A d S_{d}$.

Now let us consider the case when the conformal dimension is $\Delta=\frac{d-1}{2}$ or the term in the square root of (C.4) vanishes, we have

$$
\left.m^{2}\right|_{\mathrm{BF}}=-\left(s+\frac{d-5}{2}\right)^{2} .
$$

The mass then satisfies the Breitenlohner-Freedman bound for the spin $s$ field in $A d S_{d}$. Using (C.5) in (C.2) we obtain

$$
\left.m_{s}^{2}\right|_{\mathrm{BF}}=-s-\left(\frac{d-1}{2}\right)^{2}
$$

Note that for the scalars this condition reduces to the familiar condition [40]

$$
\left.m_{s=0}^{2}\right|_{\mathrm{BF}}=-\left(\frac{d-1}{2}\right)^{2}
$$

\footnotetext{
${ }^{14}$ Our signature for Lorentzian $A d S_{d-1}$ is $(-1,1,1, \cdots)$, and the radius of AdS is chosen to be unity.
} 
The eigenvalue of the spin- $s$ Laplacian $\Delta_{(s)}$ on Euclidean $A d S_{d}$ is given by [20]

$$
\Delta_{(s)} \psi_{\mu_{1}, \mu_{2} \cdots \mu_{s}}^{(\lambda, u)}=-\left(\lambda^{2}+s+\left(\frac{d-1}{2}\right)^{2}\right) \psi_{\mu_{1}, \mu_{2} \cdots \mu_{s}}^{(\lambda)}
$$

where $\psi_{\mu_{1}, \mu_{2} \cdots \mu_{s}}^{(\lambda, u)}$ are the corresponding eigenfunctions, $u$ are other quantum numbers characterizing the eigenfunctions. Therefore the eigenvalues of $\Delta_{(s)}-m_{s}^{2}$ is given by $-\lambda^{2}$.

$$
\left(\Delta_{(s)}-m_{s}^{2}\right) \psi_{\mu_{1}, \mu_{2} \cdots \mu_{s}}^{(\lambda, u)}=-\lambda^{2} \psi_{\mu_{1}, \mu_{2} \cdots \mu_{s}}^{(\lambda, u)} .
$$

Note that once we demand that mass of the higher spin field saturates its BreitenlohnerFreedman bound, the eigenvalues of the corresponding massive higher spin field is independent of the spin and is given by $-\lambda^{2}$.

\section{Twist weight from the replica cone: Maxwell field}

In this section we review the evaluation of the expectation value of the Maxwell stress tensor on the replica cone in $D=4$. This was originally done in [13], we adapt it so that the method can be extended for the 2-form in $D=6$. The geometry is same as that discussed in section 3.1 for the planar co-dimension 2 defect. To evaluate the expectation value of the stress tensor we use the replica trick and consider the theory on the cone. The transverse directions to the defect are labelled as $x^{a}$ with $a \in\{1,2\}$ and the longitudinal directions are labelled as $x^{i}$ with $i \in\{3,4\}$. Stress tensor for $\mathrm{U}(1)$ theory is given by

$$
\begin{aligned}
& T_{\mu \nu}=F_{\mu}{ }^{\lambda} F_{\nu \lambda}-\frac{1}{4} g_{\mu \nu} F_{\alpha \beta} F^{\alpha \beta}, \\
& F_{\mu \nu}=\partial_{\mu} A_{\nu}-\partial_{\nu} A_{\mu} .
\end{aligned}
$$

The $\mathrm{U}(1)$ theory is gauge invariant under the transformation

$$
A_{\mu} \rightarrow A_{\mu}+\partial_{\mu} \epsilon
$$

Using this symmetry we can fix the covariant gauge

$$
\partial^{\mu} A_{\mu}=0 .
$$

The equations of motion in this gauge reduces to

$$
\nabla^{2} A_{\mu}=0 .
$$

The still allows us the transformation

$$
A_{\mu}^{\prime}=A_{\mu}+\partial_{\mu} \epsilon, \quad \text { with } \square \epsilon=0 .
$$

Then given a gauge potential which satisfies (D.3) and (D.4) we can make a further gauge transformation so that

$$
\partial^{a} A_{a}^{\prime}=0, \quad \partial^{i} A_{i}^{\prime}=, 0 \quad a \in\{1,2\}, i \in\{3,4\} .
$$


This can be achieved by choosing the gauge transformation to be

$$
\epsilon=-\frac{\partial^{i} A_{i}}{\widehat{\nabla}^{2}}, \quad \widehat{\nabla}^{2}=\partial_{3}^{2}+\partial_{4}^{2} .
$$

Note that the gauge transformation also satisfies $\square \epsilon=0$.

We need to evaluate the expectation value of the stress tensor on the replica cone. For this it is convenient to choose polar coordinates

$$
d s^{2}=d r^{2}+r^{2} d \theta^{2}+\left(d x^{3}\right)^{2}+\left(d x^{4}\right)^{2} .
$$

Here $\theta \sim \theta+2 \pi q$. The two point function fo the gauge field on the cone satisfying the gauge condition (D.6), is given by

$$
\begin{aligned}
& \tilde{G}_{\mu \nu^{\prime}}\left(x, x^{\prime}\right)=\left\langle A_{\mu}(x) A_{\nu^{\prime}}\left(x^{\prime}\right)\right\rangle . \\
& \tilde{G}_{a b^{\prime}}\left(x, x^{\prime}\right)=-\frac{P_{a} P_{a^{\prime}}}{\hat{\nabla}^{2}} \tilde{G}\left(x, x^{\prime}\right), \quad \tilde{G}_{i j^{\prime}}\left(x, x^{\prime}\right)=-\left[\delta_{i j}-\frac{\partial_{i} \partial_{j}}{\widehat{\nabla}^{2}}\right] \tilde{G}\left(x, x^{\prime}\right), \\
& \tilde{G}_{a i^{\prime}}\left(x, x^{\prime}\right)=\tilde{G}_{i a^{\prime}}\left(x, x^{\prime}\right)=0 .
\end{aligned}
$$

where $\tilde{G}\left(x, x^{\prime}\right)$ is the scalar propagator on the cone which is given by

$$
\begin{aligned}
\tilde{G}\left(x, x^{\prime}\right) & =\frac{1}{4 q \pi^{2} r r^{\prime}\left(a-a^{-1}\right)} \frac{a^{\frac{1}{q}}-a^{-\frac{1}{q}}}{a^{\frac{1}{q}}+a^{-\frac{1}{q}}-2 \cos \left(\frac{\theta-\theta^{\prime}}{q}\right)}, \\
\frac{a}{1+a^{2}} & =\frac{r r^{\prime}}{\left(x^{i}-x^{\prime i}\right)^{2}+r^{2}+r^{\prime 2}} .
\end{aligned}
$$

and $P_{a}$ is defined as in (3.47). To remove the divergences that occur in the point split approach for evaluating the expectation value of the stress tensor we regulate the Greens function by considering

$$
\left.G_{\mu \nu^{\prime}}\left(x, x^{\prime}\right)=\left.\tilde{G}_{\mu \nu^{\prime}}\left(x, x^{\prime}\right)\right|_{q}-\left.\tilde{G}_{\mu \nu^{\prime}}\left(x, x^{\prime}\right)\right|_{q=1}, \quad G\left(x, x^{\prime}\right)=\left.\tilde{G}\left(x, x^{\prime}\right)\right|_{q}-\tilde{G}_{(} x, x^{\prime}\right)\left.\right|_{q=1}
$$

The expectation value of the stress tensor is then obtained by taking the following limit

$$
\left\langle T_{\mu \nu}(x) \sigma_{q}\right\rangle=\lim _{x \rightarrow x^{\prime}}\left(\left\langle F_{\mu \lambda}(x) F_{\nu}^{\lambda^{\prime}}\left(x^{\prime}\right)\right\rangle-\frac{g_{\mu \nu}}{4}\left\langle F_{\rho \sigma}(x) F^{\rho^{\prime} \sigma^{\prime}}\left(x^{\prime}\right)\right\rangle\right) .
$$

Here the two point function on the r.h.s. of the above equation are obtained by using the regularised Greens function in (D.11). This leads to 6 terms in the following equation

$$
\begin{aligned}
\left\langle T_{\mu \nu}(x) \sigma_{q}\right\rangle= & \lim _{x \rightarrow x^{\prime}}\left[\nabla_{\mu \nu^{\prime}} G_{\lambda}^{\lambda^{\prime}}\left(x, x^{\prime}\right)-\nabla_{\mu}^{\lambda^{\prime}} G_{\lambda \nu^{\prime}}\left(x, x^{\prime}\right)-\nabla_{\nu^{\prime}}^{\lambda} G_{\mu \lambda^{\prime}}\left(x, x^{\prime}\right)+\nabla_{\lambda}^{\lambda^{\prime}} G_{\mu \nu^{\prime}}\left(x, x^{\prime}\right)\right. \\
& \left.-\frac{1}{2} g_{\mu \nu^{\prime}}(x)\left(\nabla_{\mu}^{\mu^{\prime}} G_{\nu}^{\nu^{\prime}}\left(x, x^{\prime}\right)-\nabla_{\mu}^{\nu^{\prime}} G_{\nu}^{\mu^{\prime}}\left(x, x^{\prime}\right)\right)\right] .
\end{aligned}
$$

where $\nabla_{\mu \nu^{\prime}}=\nabla_{\mu} \nabla_{\nu^{\prime}}$. 
To proceed further we would need the following relation

$$
\lim _{x \rightarrow x^{\prime}} P_{a} P^{a \prime} G\left(x, x^{\prime}\right)=\lim _{x \rightarrow x^{\prime}} \nabla_{a} \nabla^{a^{\prime}} G\left(x, x^{\prime}\right) .
$$

The repeated indices always mean that we sum over them, inspite of the fact that one set of indices are labelled with a prime. We also have

$$
\begin{aligned}
\left(\nabla_{1} P_{2}-\nabla_{2} P_{1}\right) G\left(x, x^{\prime}\right) & =-r\left(\frac{\partial^{2}}{\partial r^{2}}+\frac{1}{r} \frac{\partial}{\partial r}+\frac{1}{r^{2}} \frac{\partial^{2}}{\partial \theta^{2}}\right) G\left(x, x^{\prime}\right) \\
& =r \widehat{\nabla}^{2} G\left(x, x^{\prime}\right)
\end{aligned}
$$

In the last line we have used the equation satisfied by the Scalar Greens function for when $x \neq x^{\prime} ; \square G\left(x, x^{\prime}\right)=0$. Finally

$$
\nabla^{2^{\prime}} P^{1^{\prime}}-\nabla^{1^{\prime}} P^{2^{\prime}} G\left(x, x^{\prime}\right)=-\frac{1}{r^{\prime}} \widehat{\nabla}^{2} G\left(x, x^{\prime}\right) .
$$

To arrive at this result we need to use the Christofel symbols of the polar coordinates.

Let us now analyse the stress tensor in (D.13) term by term. We first study when the external indices are both longitudinal direction to the defect. The first term is given by

$$
\begin{aligned}
\nabla_{i j^{\prime}} G_{\lambda}^{\lambda^{\prime}}\left(x, x^{\prime}\right) & =\nabla_{i j^{\prime}}\left(G_{k}^{k^{\prime}}+G_{c}^{c^{\prime}}\right) \\
& =\nabla_{i j^{\prime}}\left[-\left(\delta_{k}^{k}-\frac{\partial_{k} \partial^{k}}{\widehat{\nabla}^{2}}\right)-\frac{P_{c} P^{c^{\prime}}}{\widehat{\nabla}^{2}}\right] G\left(x, x^{\prime}\right) \\
& =-\left(1+\frac{P_{c} P^{c^{\prime}}}{\widehat{\nabla}^{2}}\right) \nabla_{i j^{\prime}} G\left(x, x^{\prime}\right), \\
& =\frac{1}{2} \delta_{i j}\left[\widehat{\nabla}^{2}+P_{a} P^{a^{\prime}}\right] G\left(x, x^{\prime}\right) .
\end{aligned}
$$

To arrive at the line of the above equation we have neglected terms which vanish on taking the $x \rightarrow x^{\prime}$ limit. More explicitly it can be seen that the following identities hold

$$
\begin{array}{r}
\lim _{x \rightarrow x^{\prime}}\left(\nabla^{2}\right)\left(\nabla_{i j^{\prime}}+\frac{\delta_{i j}}{2} \widehat{\nabla}^{2}\right) G\left(x, x^{\prime}\right)=0, \\
\lim _{x \rightarrow x^{\prime}}\left(P_{c} P^{c^{\prime}}\right)\left(\nabla_{i j^{\prime}}+\frac{\delta_{i j}}{2} \widehat{\nabla}^{2}\right) G\left(x, x^{\prime}\right)=0, \\
\lim _{x \rightarrow x^{\prime}}\left(\nabla_{c} \nabla^{c^{\prime}}\right)\left(\nabla_{i j^{\prime}}+\frac{\delta_{i j}}{2} \widehat{\nabla}^{2}\right) G\left(x, x^{\prime}\right)=0 .
\end{array}
$$

The second and the third term in (D.13) vanish since the Greens function satisfies the gauge condition (D.6) For example consider the second term

$$
-\nabla_{i}^{\lambda \prime} G_{\lambda j^{\prime}}\left(x, x^{\prime}\right)=-\nabla_{i} \nabla^{k^{\prime}} G_{k j^{\prime}}\left(x, x^{\prime}\right)=+\nabla_{i} \nabla^{k} G_{k j^{\prime}}\left(x, x^{\prime}\right)=0 .
$$

We have converted the derivative on the primed coordinate to the unprimed using translational invariance of the Greens function in the longitudinal directions. Similarly the third term in (D.13) vanishes

$$
-\nabla_{j^{\prime}}^{\lambda} G_{i \lambda^{\prime}}\left(x, x^{\prime}\right)=0
$$


Let us now look at the fourth term

$$
\begin{aligned}
+\nabla_{\lambda}^{\lambda^{\prime}} G_{i j^{\prime}}\left(x, x^{\prime}\right) & =-\left(\nabla_{k} \nabla^{k^{\prime}}+\nabla_{c} \nabla^{c^{\prime}}\right)\left(\delta_{i j}-\frac{\partial_{i} \partial_{j}}{\widehat{\nabla}^{2}}\right) G\left(x, x^{\prime}\right), \\
& =-\left(-\hat{\nabla}^{2}+\nabla_{c} \nabla^{c^{\prime}}\right)\left[\frac{1}{2} \delta_{i j}\right] G\left(x, x^{\prime}\right) .
\end{aligned}
$$

In the last line we have used the identities in (D.18) to simplify the term proportional to $\frac{\partial_{i} \partial_{j}}{\widehat{\nabla}^{2}}$. Now let us look the fifth term in (D.13).

$$
\begin{aligned}
-\frac{1}{2} \delta_{i j}\left(\nabla_{\mu}^{\mu^{\prime}} G_{\nu}^{\nu^{\prime}}\right) & =-\frac{1}{2} \delta_{i j}\left(\nabla_{k} \nabla^{k^{\prime}}+\nabla_{c} \nabla^{c^{\prime}}\right)\left[-\left(\delta_{k}^{k}-\frac{\partial_{k} \partial^{k}}{\widehat{\nabla}^{2}}\right)-\frac{P_{d} P^{d^{\prime}}}{\widehat{\nabla}^{2}}\right] G\left(x, x^{\prime}\right), \\
& =\frac{1}{2} \delta_{i j}\left(-\nabla^{2}+\nabla_{c} \nabla^{c^{\prime}}\right)\left(1+\frac{P_{d} P^{d^{\prime}}}{\widehat{\nabla}^{2}}\right) \tilde{G}\left(x, x^{\prime}\right), \\
& =\frac{1}{2} \delta_{i j}\left[-\hat{\nabla}^{2}+\frac{\nabla_{c} P_{d} \nabla^{c^{\prime}} P^{d^{\prime}}}{\widehat{\nabla}^{2}}\right] G\left(x, x^{\prime}\right) .
\end{aligned}
$$

Finally the sixth term is given by

$$
\begin{aligned}
\frac{1}{2} \delta_{i j} \nabla_{\mu}^{\nu^{\prime}} G_{\nu}^{\mu}\left(x, x^{\prime}\right) & =\frac{1}{2} \delta_{i j} \nabla_{c}^{d^{\prime}} G_{d}^{c^{\prime}}\left(x, x^{\prime}\right), \\
& =-\frac{1}{2} \delta_{i j} \frac{\nabla_{c} P_{d} \nabla^{d^{\prime}} P^{c^{\prime}}}{\hat{\nabla}^{2}} G\left(x, x^{\prime}\right) .
\end{aligned}
$$

To arrive at the first equality in the above equation we have used the fact that Green's function satisfies the gauge condition $\nabla_{i}^{l^{\prime}} G_{l}^{k^{\prime}}=0$. Let us now combine the fifth and the sixth term in (D.22) and (D.23). We obtain

$$
\begin{aligned}
\frac{1}{2} \delta_{i j}\left[-\hat{\nabla}^{2}\right. & \left.+\frac{\nabla_{c} P_{d}\left(\nabla^{c^{\prime}} P^{d^{\prime}}-\nabla^{d^{\prime}} P^{c^{\prime}}\right)}{\widehat{\nabla}^{2}}\right] G\left(x, x^{\prime}\right), \\
& =\frac{1}{2} \delta_{i j}\left[-\hat{\nabla}^{2}+\frac{\left(\nabla_{1} P_{2}-\nabla_{2} P_{1}\right)\left(\nabla^{1^{\prime}} P^{2^{\prime}}-\nabla^{2^{\prime}} P^{1^{\prime}}\right)}{\hat{\nabla}^{2}}\right] G\left(x, x^{\prime}\right), \\
& =\frac{1}{2} \delta_{i j}\left[-\hat{\nabla}^{2}+\hat{\nabla}^{2}\right] G\left(x, x^{\prime}\right)=0 .
\end{aligned}
$$

The last line is obtained by using the identities in (D.15) and (D.16) and also set $r=r^{\prime}$ external to the differential operators. We have shown that adding the 5 th and the 6 th term vanishes. From the origin of these terms we conclude

$$
\lim _{x \rightarrow x^{\prime}}\left\langle F_{\mu \nu}(x) F^{\mu^{\prime} \nu^{\prime}}\left(x^{\prime}\right) \sigma_{q}\right\rangle=0 .
$$

What remains therefore are the 1st and the 4th terms. Combining (D.17) and (D.19) and using (D.14) we conclude that

$$
\left\langle T_{i j}(x)\right\rangle=\delta_{i j} \lim _{x \rightarrow x^{\prime}} \hat{\nabla}^{2} G\left(x, x^{\prime}\right)
$$


Let us now examine the transverse components of the stress tensor, we chose the free indices to be $a, b$. From (D.13), we see that the first term is given by

$$
\begin{aligned}
\nabla_{a} \nabla^{b^{\prime}} G_{\lambda}^{\lambda^{\prime}}\left(x, x^{\prime}\right) & =\nabla_{a} \nabla^{b^{\prime}}\left(G_{k}^{k^{\prime}}\left(x, x^{\prime}\right)+G_{c}^{c^{\prime}}\left(x, x^{\prime}\right)\right), \\
& =\nabla_{a} \nabla^{b^{\prime}}\left(-1-\frac{P_{c} P^{c^{\prime}}}{\widehat{\nabla}^{2}}\right) G\left(x, x^{\prime}\right), \\
& =\left(-\nabla_{a} \nabla^{b^{\prime}}-\frac{\nabla_{a} P_{c} \nabla^{b^{\prime}} P^{c^{\prime}}}{\hat{\nabla}^{2}}\right) G\left(x, x^{\prime}\right) .
\end{aligned}
$$

Expanding the second term we get

$$
-\nabla_{a}^{\lambda^{\prime}} G_{\lambda}^{b^{\prime}}\left(x, x^{\prime}\right)=\frac{\nabla_{a} P_{c} \nabla^{c^{\prime}} P^{b^{\prime}}}{\widehat{\nabla}^{2}} G\left(x, x^{\prime}\right)
$$

Similarly the third term of (D.13) for the transverse components becomes

$$
-\nabla^{b^{\prime}} \nabla^{\lambda} G_{a \lambda^{\prime}}\left(x, x^{\prime}\right)=\frac{\nabla_{c} P_{a} \nabla^{b^{\prime}} P^{c^{\prime}}}{\widehat{\nabla}^{2}} G\left(x, x^{\prime}\right) .
$$

The fourth term results in

$$
\begin{aligned}
\nabla_{\lambda}^{\lambda^{\prime}} G_{a}^{b^{\prime}}\left(x, x^{\prime}\right) & =\left(-\nabla^{2}+\nabla_{c} \nabla^{c^{\prime}}\left(\frac{-P_{a} P^{b^{\prime}}}{\widehat{\nabla}^{2}}\right)\right) G\left(x, x^{\prime}\right), \\
& =\left(P_{a} P^{b^{\prime}}-\frac{\nabla_{c} P_{a} \nabla^{c^{\prime}} P^{b^{\prime}}}{\widehat{\nabla}^{2}}\right) G\left(x, x^{\prime}\right) .
\end{aligned}
$$

The fifth and the sixth term together vanishes due to (D.25). Combining (D.27), (D.28), (D.29) and (D.30) we obtain

$$
\begin{aligned}
\left\langle T_{a}^{b}(x) \sigma_{q}\right\rangle & =\lim _{x \rightarrow x^{\prime}}\left[-\nabla_{a} \nabla^{b^{\prime}}+P_{a} P^{b^{\prime}}+\frac{\left(\nabla_{a} P_{c}-\nabla_{c} P_{a}\right)\left(\nabla^{c^{\prime}} P^{b^{\prime}}-\nabla^{b^{\prime}} P^{c^{\prime}}\right)}{\hat{\nabla}^{2}}\right] G\left(x, x^{\prime}\right), \\
& =\lim _{x \rightarrow x^{\prime}}\left(-\nabla_{a} \nabla^{b^{\prime}}+P_{a} P^{b^{\prime}}-\delta_{a}^{b} \widehat{\nabla}^{2}\right) G\left(x, x^{\prime}\right) .
\end{aligned}
$$

To arrive at the last line we have used the identities in (D.15) and (D.16). We write down the non-trivial components explicitly

$$
\begin{aligned}
\left\langle T_{r}^{r}(x) \sigma_{q}\right\rangle & =\lim _{x \rightarrow x^{\prime}}\left(-\partial_{r} \partial_{r^{\prime}}+\frac{1}{r^{2}} \partial_{\theta} \partial_{\theta^{\prime}}-\hat{\nabla}^{2}\right) G\left(x, x^{\prime}\right), \\
& =\frac{\left(q^{2}-1\right)\left(11 q^{2}+1\right)}{720 \pi^{2} q^{4} r^{4}}, \\
\left\langle T_{\theta}^{\theta}(x) \sigma_{q}\right\rangle & =\lim _{x \rightarrow x^{\prime}}\left(\partial_{r} \partial_{r^{\prime}}-\frac{1}{r^{2}} \partial_{\theta} \partial_{\theta^{\prime}}-\hat{\nabla}^{2}\right) G\left(x, x^{\prime}\right), \\
& =\frac{-11 q^{4}+10 q^{2}+1}{240 \pi^{2} q^{4} r^{4}} .
\end{aligned}
$$


Transforming these components to cartesian coordinates we get

$$
\begin{aligned}
\left\langle T_{11}(x) \sigma_{n}\right\rangle & =\frac{\left(q^{2}-1\right)\left(11 q^{2}+1\right)}{720 \pi^{2} q^{4}} \frac{\left(x_{1}^{2}-3 x_{2}^{2}\right)}{r^{6}}, \\
\left\langle T_{22}(x) \sigma_{n}\right\rangle & =\frac{\left(q^{2}-1\right)\left(11 q^{2}+1\right)}{720 \pi^{2} q^{4}} \frac{\left(x_{2}^{2}-3 x_{1}^{2}\right)}{r^{6}}, \\
\left\langle T_{i j}(x) \sigma_{n}\right\rangle & =-\delta_{i j} \frac{\left(q^{2}-1\right)\left(11 q^{2}+1\right)}{720 \pi^{2} q^{4} r^{4}} .
\end{aligned}
$$

The last line results from taking the limit in (D.26). Now comparing the equations for the expectation value of the stress tensor in presence of the co-dimension 2 defect in (3.35) we can read out the value of its conformal dimension

$$
h_{q}=\frac{\left(q^{2}-1\right)\left(11 q^{2}+1\right)}{360 \pi q^{3}} .
$$

\section{E Rényi entropies and weights of twists}

Symmetric tensors. We list the Rényi entropies and the conformal dimension of the co-dimensions two twist operator corresponding to the spherical entangling surface for all $4 \leq D \leq 14$ for symmetric rank-s tensors.

- $D=4$ :

$$
\begin{aligned}
& S_{q}=\frac{(q+1)\left[\left(30 s^{2}+1\right) q^{2}+1\right]}{180 q^{3}} \log \epsilon, \\
& h_{q}=\frac{\left(q^{2}-1\right)\left(q^{2}\left(10 s^{2}+1\right)+1\right)}{360 \pi q^{3}} .
\end{aligned}
$$

- $D=6$ :

$$
\begin{aligned}
& S_{q}=-\frac{(q+1)(s+1)^{2}\left(\left(q^{2}+1\right) q^{2}(7 s(s+2)+9)+2\right)}{30240 q^{5} s !} \log (\epsilon), \\
& h_{q}=\frac{(s+1)^{2}\left(q^{6}(21 s(s+2)+31)-21 q^{2}(s+1)^{2}-10\right)}{151200 \pi^{2} q^{5} s !} .
\end{aligned}
$$

- $D=8$ :

$$
\begin{aligned}
& S_{q}=\frac{(s+2)(s+3) !}{21772800 q^{7}(1-q) s !}\left[q^{8}(-(18 s(s+4)+79))\right. \\
&+\left.14 q^{4}(s+2)^{2}+4 q^{2}(s(s+4)+5)+3\right] \log (\epsilon), \\
& h_{q}=\frac{\left(q^{2}-1\right)(s+1)(s+2)^{2}(s+3)}{50803200 \pi^{3} q^{7}}\left[q^{6}(62 s(s+4)+289)\right. \\
&\left.+q^{4}(62 s(s+4)+289)+q^{2}(20 s(s+4)+121)+21\right] .
\end{aligned}
$$


- $D=10$ :

$$
\begin{aligned}
& S_{q}=\frac{-(s+3)(s+5) !}{1207084032000 q^{9}(1-q) s !}\left[q^{10}(-(2607 s(s+6)+24626))\right. \\
& +1848 q^{6}(s+3)^{2}+132 q^{4}(5 s(s+6)+49) \\
& \left.+99 q^{2}(s(s+6)+14)+140\right] \log (\epsilon), \\
& h_{q}=\frac{\left(q^{2}-1\right)(s+3) \Gamma(s+6)}{301771008000 \pi^{4} q^{9} s !}\left[q^{8}(3179 s(s+6)+31066)\right. \\
& +q^{6}(3179 s(s+6)+31066)+q^{4}(1331 s(s+6)+14434) \\
& \left.+21 q^{2}(11 s(s+6)+174)+420\right] \text {. }
\end{aligned}
$$

- $D=12$ :

$$
\begin{aligned}
& S_{q}=\frac{(s+4)(s+7) !}{79088145776640000 q^{11}(1-q) s !}\left[q^{12}(-(320138 s(s+8)+5276673))\right. \\
& +216216 q^{8}(s+4)^{2}+1716 q^{6}(49 s(s+8)+820) \\
& \left.+9009 q^{4}(2 s(s+8)+39)+1820 q^{2}(s(s+8)+30)+4146\right] \log (\epsilon), \\
& h_{q}=\frac{\Gamma(s+8)\left(q^{2}-1\right)(s+4)}{4833164464128000 \pi^{5} q^{12} s !}\left[q ^ { 2 } \left(q^{8}(403858 s(s+8)+6803477)\right.\right. \\
& +q^{6}(403858 s(s+8)+6803477)+q^{4}(187642 s(s+8)+3344021) \\
& \left.\left.+q^{2}(47502 s(s+8)+998821)+5460 s(s+8)+179002\right)+15202\right] \text {. }
\end{aligned}
$$

- $D=14$ :

$$
\begin{aligned}
& S_{q}=\frac{-(s+5)(s+9) !}{313189057275494400000 q^{13}(1-q) s !}\left[q^{14}(-(1758891 s(s+10)+44865227))\right. \\
& +1153152 q^{10}(s+5)^{2}+2288 q^{8}(205 s(s+10)+5269) \\
& +429 q^{6}(273 s(s+10)+7645)+1820 q^{4}(10 s(s+10)+341) \\
& \left.+1382 q^{2}(s(s+10)+55)+4620\right] \log (\epsilon), \\
& h_{q}=\frac{\left(q^{2}-1\right)(s+5)(s+9) !}{11309604846059520000 \pi^{6} q^{13} s !}\left[q^{12}(6803477 s(s+10)+176203445)\right. \\
& +q^{10}(6803477 s(s+10)+176203445) \\
& +q^{8}(3344021 s(s+10)+89717045) \\
& +q^{6}(998821 s(s+10)+29439685) \\
& +2 q^{4}(89501 s(s+10)+3240875) \\
& \left.+22 q^{2}(691 s(s+10)+40735)+60060\right] \text {. }
\end{aligned}
$$

We have verified that the above results satisfy the relation between the derivatives of $S_{q}$ and $h_{q}$ found in [14]. The relation between the derivatives on $S_{q}$ and $h_{q}$ at $q=1$ is 
given by

$$
\left.\partial_{q} S_{q}\right|_{q=1}=-\left.\frac{d \operatorname{Vol}\left(A d S_{d}\right)}{2} \partial_{q} h_{q}\right|_{q=1}
$$

For example in $D=6$ we see that

$$
\begin{aligned}
\left.\partial_{q} S_{q}\right|_{q=1} & =-\left.\frac{5 \operatorname{Vol}\left(A d S_{d}\right)}{2} \partial_{q} h_{q}\right|_{q=1}, \\
& =\frac{(s+1)^{2}(7 s(s+2)+12)}{5040 s !} \log \epsilon .
\end{aligned}
$$

The relation (E.13) has been verified for all the listed values of $S_{q}$ and $h_{q}$.

$\boldsymbol{p}$-forms. We list the Rényi entropies and the conformal dimensions of the co-dimension 2 twist operators for conformal forms in even $D$ dimensions. The conformal form is the $D / 2$ - 1 -form.

- $D=4: 1$-form

$$
\begin{aligned}
& S_{q}=\frac{(q+1)\left(31 q^{2}+1\right)}{180 q^{3}} \log (\epsilon) . \\
& h_{q}=\frac{\left(q^{2}-1\right)\left(11 q^{2}+1\right)}{360 \pi q^{3}} .
\end{aligned}
$$

- $D=6: 2$-form

$$
\begin{gathered}
S_{q}=-\frac{(q+1)\left(877 q^{4}+37 q^{2}+2\right)}{5040 q^{5}} \log (\epsilon), \\
h_{q}=\frac{(q-1)(q+1)\left(191 q^{4}+23 q^{2}+2\right)}{5040 \pi^{2} q^{5}} .
\end{gathered}
$$

- $D=8: 3$-form

$$
\begin{aligned}
& S_{q}=\frac{(q+1)\left(15865 q^{6}+745 q^{4}+59 q^{2}+3\right)}{90720 q^{7}} \log (\epsilon), \\
& h_{q}=\frac{(q-1)(q+1)\left(11 q^{2}+1\right)\left(227 q^{4}+10 q^{2}+3\right)}{30240 \pi^{3} q^{7}} .
\end{aligned}
$$

- $D=10: 4$-form

$$
\begin{aligned}
& S_{q}=-\frac{(q+1)\left(4200673 q^{8}+208993 q^{6}+19573 q^{4}+1555 q^{2}+70\right)}{23950080 q^{9}} \log (\epsilon), \\
& h_{q}=\frac{(q-1)(q+1)\left(14797 q^{8}+2125 q^{6}+321 q^{4}+35 q^{2}+2\right)}{57024 \pi^{4} q^{9}} .
\end{aligned}
$$

- $D=12: 5$-form

$$
\begin{gathered}
S_{q}=\frac{(q+1)}{15567552000 q^{11}}\left[2735714123 q^{10}+141122123 q^{8}+14539667 q^{6}\right. \\
\left.+1420847 q^{4}+104246 q^{2}+4146\right] \log (\epsilon), \\
h_{q}=\frac{\left(q^{2}-1\right)}{86486400 \pi^{5} q^{11}}\left[\left(92427157 q^{10}+13803157 q^{8}+2295661 q^{6}\right.\right. \\
\left.+307961 q^{4}+28682 q^{2}+1382\right] .
\end{gathered}
$$


- $D=14: 6$-form

$$
\begin{gathered}
S_{q}=-\frac{(q+1)}{186810624000 q^{13}}\left[32873434703 q^{12}+1738330703 q^{10}+190512431 q^{8}\right. \\
\left.+21031119 q^{6}+1952202 q^{4}+130382 q^{2}+4620\right] \log (\epsilon), \\
h_{q}=\frac{(q-1)(q+1)}{6739200 \pi^{6} q^{13}}\left[36740617 q^{12}+5636617 q^{10}+997801 q^{8}\right. \\
\left.+151241 q^{6}+17822 q^{4}+1442 q^{2}+60\right] .
\end{gathered}
$$

Open Access. This article is distributed under the terms of the Creative Commons Attribution License (CC-BY 4.0), which permits any use, distribution and reproduction in any medium, provided the original author(s) and source are credited.

\section{References}

[1] V. Benedetti and H. Casini, Entanglement entropy of linearized gravitons in a sphere, Phys. Rev. D 101 (2020) 045004 [arXiv: 1908.01800] [INSPIRE].

[2] W. Donnelly and A.C. Wall, Entanglement entropy of electromagnetic edge modes, Phys. Rev. Lett. 114 (2015) 111603 [arXiv:1412.1895] [INSPIRE].

[3] K.-W. Huang, Central charge and entangled gauge fields, Phys. Rev. D 92 (2015) 025010 [arXiv:1412.2730] [INSPIRE].

[4] W. Donnelly and A.C. Wall, Geometric entropy and edge modes of the electromagnetic field, Phys. Rev. D 94 (2016) 104053 [arXiv:1506.05792] [INSPIRE].

[5] R.M. Soni and S.P. Trivedi, Entanglement entropy in $(3+1)-d$ free U(1) gauge theory, JHEP 02 (2017) 101 [arXiv: 1608.00353] [INSPIRE].

[6] U. Moitra, R.M. Soni and S.P. Trivedi, Entanglement entropy, relative entropy and duality, JHEP 08 (2019) 059 [arXiv: 1811. 06986] [inSPIRE].

[7] J.S. Dowker, Note on the entanglement entropy of higher spins in four dimensions, arXiv: 1908.04870 [INSPIRE].

[8] J.S. Dowker, Arbitrary spin theory in the Einstein universe, Phys. Rev. D 28 (1983) 3013 [INSPIRE].

[9] H. Casini, M. Huerta and R.C. Myers, Towards a derivation of holographic entanglement entropy, JHEP 05 (2011) 036 [arXiv:1102.0440] [INSPIRE].

[10] E.S. Fradkin and A.A. Tseytlin, One loop effective potential in gauged $O(4)$ supergravity, Nucl. Phys. B 234 (1984) 472 [InSPIRE].

[11] J.R. David, M.R. Gaberdiel and R. Gopakumar, The heat kernel on $A d S_{3}$ and its applications, JHEP 04 (2010) 125 [arXiv:0911.5085] [INSPIRE].

[12] M.R. Gaberdiel, R. Gopakumar and A. Saha, Quantum W-symmetry in AdS $S_{3}$, JHEP 02 (2011) 004 [arXiv: 1009.6087] [INSPIRE].

[13] P. Candelas and D. Deutsch, On the vacuum stress induced by uniform acceleration or supporting the ether, Proc. Roy. Soc. Lond. A 354 (1977) 79. 
[14] L.-Y. Hung, R.C. Myers and M. Smolkin, Twist operators in higher dimensions, JHEP 10 (2014) 178 [arXiv: 1407.6429] [INSPIRE].

[15] D. Anninos, F. Denef, Y.T.A. Law and Z. Sun, Quantum de Sitter horizon entropy from quasicanonical bulk, edge, sphere and topological string partition functions, arXiv:2009.12464 [INSPIRE].

[16] L.-Y. Hung, R.C. Myers, M. Smolkin and A. Yale, Holographic calculations of Renyi entropy, JHEP 12 (2011) 047 [arXiv: 1110.1084] [INSPIRE].

[17] H. Casini and M. Huerta, Entanglement entropy for the n-sphere, Phys. Lett. B 694 (2011) 167 [arXiv: 1007.1813] [INSPIRE].

[18] C.P. Herzog and T. Nishioka, The edge of entanglement: getting the boundary right for non-minimally coupled scalar fields, JHEP 12 (2016) 138 [arXiv:1610.02261] [INSPIRE].

[19] I.R. Klebanov, S.S. Pufu, S. Sachdev and B.R. Safdi, Renyi entropies for free field theories, JHEP 04 (2012) 074 [arXiv:1111.6290] [INSPIRE].

[20] R. Camporesi and A. Higuchi, Spectral functions and zeta functions in hyperbolic spaces, $J$. Math. Phys. 35 (1994) 4217 [InSPIRE].

[21] J.S. Dowker, Vacuum averages for arbitrary spin around a cosmic string, Phys. Rev. D 36 (1987) 3742 [INSPIRE].

[22] J.S. Dowker, Entanglement entropy for even spheres, arXiv:1009.3854 [INSPIRE].

[23] C. Eling, Y. Oz and S. Theisen, Entanglement and thermal entropy of gauge fields, JHEP 11 (2013) 019 [arXiv: 1308.4964] [INSPIRE].

[24] H. Casini and M. Huerta, Entanglement entropy of a Maxwell field on the sphere, Phys. Rev. D 93 (2016) 105031 [arXiv: 1512.06182] [INSPIRE].

[25] H. Casini, M. Huerta, J.M. Magán and D. Pontello, Logarithmic coefficient of the entanglement entropy of a Maxwell field, Phys. Rev. D 101 (2020) 065020 [arXiv: 1911.00529] [INSPIRE].

[26] R. Gopakumar, R.K. Gupta and S. Lal, The heat kernel on AdS, JHEP 11 (2011) 010 [arXiv: 1103.3627] [INSPIRE].

[27] H. Osborn and A.C. Petkou, Implications of conformal invariance in field theories for general dimensions, Annals Phys. 231 (1994) 311 [hep-th/9307010] [INSPIRE].

[28] J.S. Dowker, Renyi entropy and $C_{T}$ for $p$-forms on even spheres, arXiv:1706.04574 [INSPIRE].

[29] J. Nian and Y. Zhou, Rényi entropy of a free $(2,0)$ tensor multiplet and its supersymmetric counterpart, Phys. Rev. D 93 (2016) 125010 [arXiv:1511.00313] [INSPIRE].

[30] R. Camporesi and A. Higuchi, The Plancherel measure for p-forms in real hyperbolic spaces, J. Geom. Phys. 15 (1994) 57.

[31] E. Witten, Anti-de Sitter space and holography, Adv. Theor. Math. Phys. 2 (1998) 253 [hep-th/9802150] [INSPIRE].

[32] M. Nozaki, T. Numasawa and T. Takayanagi, Quantum entanglement of local operators in conformal field theories, Phys. Rev. Lett. 112 (2014) 111602 [arXiv:1401.0539] [inSPIRE].

[33] J. Lee, A. Lewkowycz, E. Perlmutter and B.R. Safdi, Rényi entropy, stationarity, and entanglement of the conformal scalar, JHEP 03 (2015) 075 [arXiv:1407.7816] [INSPIRE]. 
[34] A. Buchel, J. Escobedo, R.C. Myers, M.F. Paulos, A. Sinha and M. Smolkin, Holographic GB gravity in arbitrary dimensions, JHEP 03 (2010) 111 [arXiv:0911.4257] [INSPIRE].

[35] I.V. Tyutin and M.A. Vasiliev, Lagrangian formulation of irreducible massive fields of arbitrary spin in (2+1)-dimensions, Teor. Mat. Fiz. 113N1 (1997) 45 [hep-th/9704132] [INSPIRE].

[36] S. Datta and J.R. David, Higher spin quasinormal modes and one-loop determinants in the BTZ black hole, JHEP 03 (2012) 079 [arXiv:1112.4619] [INSPIRE].

[37] M. Beccaria and A.A. Tseytlin, $C_{T}$ for conformal higher spin fields from partition function on conically deformed sphere, JHEP 09 (2017) 123 [arXiv: 1707.02456] [INSPIRE].

[38] R.R. Metsaev, Massive totally symmetric fields in AdS $S_{d}$, Phys. Lett. B 590 (2004) 95 [hep-th/0312297] [INSPIRE].

[39] I.L. Buchbinder, V.A. Krykhtin and P.M. Lavrov, Gauge invariant Lagrangian formulation of higher spin massive bosonic field theory in AdS space, Nucl. Phys. B 762 (2007) 344 [hep-th/0608005] [INSPIRE].

[40] P. Breitenlohner and D.Z. Freedman, Stability in gauged extended supergravity, Annals Phys. 144 (1982) 249 [INSPIRE]. 